Mon. Not. R. Astron. Soc. 000, 000-000 (0000) Printed 29 May $2022 \quad$ (MN LATEX style file v2.2)

\title{
Simulating the formation of massive seed black holes in the early Universe. II: Impact of rate coefficient uncertainties
}

\author{
Simon C. O. Glover \\ Universität Heidelberg, Zentrum für Astronomie, Institut für Theoretische Astrophysik, \\ Albert-Ueberle-Straße 2, 69120 Heidelberg, Germany
}

29 May 2022

\begin{abstract}
We investigate how uncertainties in the chemical and cooling rate coefficients relevant for a metal-free gas influence our ability to determine the critical ultraviolet field strength required to suppress $\mathrm{H}_{2}$ cooling in high-redshift atomic cooling halos. The suppression of $\mathrm{H}_{2}$ cooling is a necessary prerequisite for the gas to undergo direct collapse and form an intermediate mass black hole. These black holes can then act as seeds for the growth of the supermassive black holes (SMBHs) observed at redshifts $z \sim$ 6 . The viability of this model for SMBH formation depends on the critical ultraviolet field strength, $J_{\text {crit }}$ : if this is too large, then too few seeds will form to explain the observed number density of SMBHs. We show in this paper that there are five key chemical reactions whose rate coefficients are uncertain enough to significantly affect $J_{\text {crit }}$. The most important of these is the collisional ionization of hydrogen by collisions with other hydrogen atoms, as the rate for this process is very poorly constrained at the low energies relevant for direct collapse. The total uncertainty introduced into $J_{\text {crit }}$ by this and the other four reactions could in the worst case approach a factor of five. We also show that the use of outdated or inappropriate values for the rates of some chemical reactions in previous studies of the direct collapse mechanism may have significantly affected the values of $J_{\text {crit }}$ determined by these studies.
\end{abstract}

Key words: astrochemistry - hydrodynamics - methods: numerical - molecular processes - cosmology: theory - quasars: general

\section{INTRODUCTION}

Observations of quasars at redshifts $z>6$ demonstrate that supermassive black holes (SMBHs) with masses of order $10^{9} \mathrm{M}_{\odot}$ had already managed to form by the time that the Universe was one billion years old. However, this is difficult to understand if these SMBHs form from the seed black holes with masses $M \sim 10 \mathrm{M}_{\odot}$ that are produced as the endpoints of the evolution of massive stars. The problem is one of timescales: even if we assume that accretion onto these seed black holes is very efficient, the time required for one of them to grow to a mass of $10^{9} \mathrm{M}_{\odot}$ can easily exceed the age of the Universe at $z>6$ (Tanaka \& Haiman 2009).

For this reason, an alternative model for the formation of the progenitors of high-redshift SMBHs has recently attracted considerable interest. In this model, known as the direct collapse model, gas in a protogalaxy with a virial temperature $T_{\text {vir }}>10^{4} \mathrm{~K}$ that is illuminated by an extremely strong extragalactic radiation field collapses quasiisothermally, maintaining its temperature in the range $T \sim$ 5000-10000 K through the combined effects of atomic hydrogen cooling and $\mathrm{H}^{-}$bound-free cooling (Omukai 2001). The high temperature keeps the Jeans mass in the collapsing gas high and inhibits fragmentation during the collapse (see e.g. Bromm \& Loeb 2003, Begelman, Volonteri, \& Rees 2006. Shang, Bryan \& Haiman 2010). The precise outcome of the collapse remains uncertain, but the most likely outcomes are either the formation of a short-lived supermassive star or a quasi-star, a massive, optically-thick core whose centre has already collapsed to form a black hole (Begelman, Volonteri, \& Rees 2006, Begelman 2010, Schleicher et al. 2013). In either case, the end result is the formation of an intermediate mass black hole $(\mathrm{IMBH})$ with a mass $M \sim 10^{4} \mathrm{M}_{\odot}$ or larger.

The time required for this much larger seed black hole to grow by accretion into a $10^{9} \mathrm{M}_{\odot} \mathrm{SMBH}$ is short enough that this mechanism can explain the existence of the observed high redshift SMBHs. In addition, the strength of the extragalactic radiation field required to suppress $\mathrm{H}_{2}$ formation and the associated cooling in the collapsing gas is much larger than the mean value produced by early stellar populations (Haiman, Abel \& Rees 2000, Greif \& Bromm 2006). This means that the formation of an IMBH by direct collapse will be a rare event, occurring only in a small number of protogalaxies located in highly clustered regions 
where the local radiation field strength can be orders of magnitude above the mean value (Dijkstra et al. 2008, Ahn et al. 2009 Agarwal et al. 2012). The viability of the direct collapse model depends upon just how rare this outcome is, and hence on how strong the radiation field needs to be in order to sufficiently suppress $\mathrm{H}_{2}$ formation. If direct collapse occurs too rarely, then not enough IMBH progenitors will be produced to explain the observed number density of SMBHs at high redshift.

Unfortunately, there is little agreement on the precise value of the radiation field strength required. This is usually quantified in terms of the specific intensity of the ultraviolet portion of the radiation field at the Lyman limit. Following Haiman, Abel \& Rees (2000), it is common to measure this in units of $10^{-21} \mathrm{ergs}^{-1} \mathrm{~cm}^{-2} \mathrm{~Hz}^{-1} \mathrm{sr}^{-1}$ and to write it as $J_{21}$. The minimum value of $J_{21}$ for which $\mathrm{H}_{2}$ cooling is sufficiently suppressed is then commonly denoted as $J_{\text {crit }}$. Physically, the value of $J_{\text {crit }}$ depends strongly on the shape of the spectrum of the extragalactic radiation field (Shang, Bryan \& Haiman 2010, Sugimura, Omukai \& Inoue 2014. Latif et al. 2015, Agarwal \& Khochfar 2015; Agarwal et al. 2015) and on the strength of any extragalactic X-ray background or cosmic ray background (Inayoshi \& Omukai 2011. Inayoshi \& Tanaka 2015, Latif et al.|2015). However, our ability to determine $J_{\text {crit }}$ accurately from simulations is also affected by uncertainties arising from the way in which the chemistry of $\mathrm{H}_{2}$ is modelled in these simulations.

There are three main ways in which the chemical model adopted can affect $J_{\text {crit }}$. First, its value in some circumstances is highly sensitive to the way in which the effects of $\mathrm{H}_{2}$ self-shielding are modelled (Wolcott-Green \& Haiman 2011, Sugimura, Omukai \& Inoue 2014). Second, for reasons of computational efficiency, it is common to model the chemistry of the gas using a highly simplified chemical network, but if the model is made too simple, then errors of up to a factor of a few can be introduced into $J_{\text {crit }}$ (Glover 2015; hereafter, Paper I). Finally, as we explore in this paper, $J_{\text {crit }}$ is also sensitive to the values adopted for the rate coefficients of some of the key chemical reactions occurring in the gas.

Since most chemical rate coefficients are not known absolutely precisely, uncertainties in the input rate coefficients inevitably introduce an uncertainty into the value of $J_{\text {crit }}$ predicted by the simulations. In this study, we attempt to quantify the size of this uncertainty and to identify which chemical reactions make the largest contributions to it. In addition, as many previous numerical studies of the direct collapse model have adopted values for some of the chemical rate coefficients that are now known to differ significantly from the actual values, we also explore how sensitive $J_{\text {crit }}$ is to these choices.

The structure of our paper is as follows. In Section 2, we briefly present the numerical method we use in our study. In Section 3, we quantify the sensitivity of $J_{\text {crit }}$ to the rate of each of the chemical reactions included in our model. Using this information, we identify a subset of reactions for which the sensitivity is large and critically discuss the uncertainties that exist in the rate coefficients for each of these reactions. In Section 4, we carry out a similar analysis for the different cooling processes included in our model. In Section 5 we combine our results and attempt to estimate the overall uncertainty in $J_{\text {crit }}$ due to uncertainties in the chemical and cooling rate coefficients. We also compare the results of our analysis with those of previous studies of the direct collapse model. Finally, we summarize the main results of our paper in Section 6 .

\section{NUMERICAL METHOD}

\subsection{The one-zone model}

We model the thermal and chemical evolution of gravitationally collapsing gas in halos illuminated by a strong extragalactic radiation field using the same one-zone model as described in detail in Paper I. We assume that the gas density evolves as

$\frac{\mathrm{d} \rho}{\mathrm{d} t}=\frac{\rho}{t_{\mathrm{ff}}}$,

where $t_{\mathrm{ff}}=(3 \pi / 32 G \rho)^{1 / 2}$ is the gravitational free-fall time. The thermal evolution of the gas is followed by solving the internal energy equation

$\frac{\mathrm{d} e}{\mathrm{~d} t}=\frac{p}{\rho^{2}} \frac{\mathrm{d} \rho}{\mathrm{d} t}+\Gamma-\Lambda$,

where $e$ is the internal energy density, $p=(\gamma-1) e$ is the gas pressure, $\Gamma$ is the radiative and chemical heating rate per unit volume and $\Lambda$ is the radiative and chemical cooling rate per unit volume. These are computed using the detailed atomic and molecular cooling function introduced in Glover \& Savin (2009) and updated in Paper I.

To model the chemical evolution of the gas, we use the conservative reduced network derived in Paper I. This consists of 25 reactions involving 8 different chemical species: $\mathrm{e}^{-}, \mathrm{H}^{+}, \mathrm{H}, \mathrm{H}^{-}, \mathrm{H}_{2}^{+}, \mathrm{H}_{2}$, He and $\mathrm{HeH}^{+}$. In Paper I we showed that the difference between the value of $J_{\text {crit }}$ derived using this network and that derived using a comprehensive chemical model of primordial gas is around 1\%, much smaller than the uncertainties considered in this paper. The full list of reactions included in our model is given in Table 1 .

We model the effects of the external ultraviolet background using two different spectral shapes: a $10^{5} \mathrm{~K}$ blackbody, cut off at $h \nu>13.6 \mathrm{eV}$ to account for absorption by neutral hydrogen, and a $10^{4} \mathrm{~K}$ black-body cut off at the same energy. For brevity, we refer to these two spectral shapes in the remainder of the paper as $\mathrm{T} 5$ and $\mathrm{T} 4$ spectra, respectively. The normalization of the radiation field is specified in terms of its specific intensity at the Lyman limit, measured in units of $10^{-21} \mathrm{ergs}^{-1} \mathrm{~cm}^{-2} \mathrm{~Hz}^{-1} \mathrm{sr}^{-1}$, and written as $J_{21}$.

We assume that the gravitationally collapsing gas is optically thin in the continuum and model the effects of $\mathrm{H}_{2}$ self-shielding using the modified version of the Draine \& Bertoldi (1996) shielding function given in Wolcott-Green, Haiman \& Bryan (2011):

$$
\begin{aligned}
f_{\mathrm{sh}}\left(N_{\mathrm{H}_{2}}, T\right)= & \frac{0.965}{\left(1+x / b_{5}\right)^{1.1}}+\frac{0.035}{(1+x)^{0.5}} \\
& \times \exp \left[-8.5 \times 10^{-4}(1+x)^{0.5}\right],
\end{aligned}
$$

where $x=N_{\mathrm{H}_{2}} / 5 \times 10^{14} \mathrm{~cm}^{-2}, b_{5}=b / 10^{5} \mathrm{~cm} \mathrm{~s}^{-1}$, and $b$ is the Doppler broadening parameter, which we assume to be dominated by the effects of thermal broadening. To compute $N_{\mathrm{H}_{2}}$, we assume that the dominant contribution comes from within a central dense core with radius equal to the Jeans length, and hence estimate $N_{\mathrm{H}_{2}}$ as $N_{\mathrm{H}_{2}}=n_{\mathrm{H}_{2}} \lambda_{\mathrm{J}}$, where $\lambda_{\mathrm{J}}$ is the Jeans length. 
As in Paper I, we caution that the simple approximation used here to compute $N_{\mathrm{H}_{2}}$ may yield values that are systematically incorrect by up to an order of magnitude. The absolute values derived here for $J_{\text {crit }}$ should therefore be treated with caution. However, this systematic uncertainty should not affect the conclusions we draw here regarding the sensitivity of $J_{\text {crit }}$ to uncertainties in the chemical rate coefficient adopted in the model.

\section{$2.2 \quad$ Initial conditions}

In paper I, we found, in common with previous authors, that the value of $J_{\text {crit }}$ depended only very weakly on our choices for the initial temperature, density and ionization state of the gas. The reason for this lack of sensitivity is that the question of whether or not enough $\mathrm{H}_{2}$ forms in the gas to provide efficient cooling to temperatures $T \ll 10^{4} \mathrm{~K}$ is generally decided once the gas has already reached a number density of $100-1000 \mathrm{~cm}^{-3}$, by which time it retains only a weak memory of its initial conditions (see e.g. Shang, Bryan \& Haiman 2010 or Paper I).

Therefore, in this study we consider runs performed using only a single set of initial conditions. We set the initial temperature to $T_{0}=8000 \mathrm{~K}$, the initial hydrogen nuclei number density to $n_{0}=0.3 \mathrm{~cm}^{-3}$, and assume that the initial fractional ionization and $\mathrm{H}_{2}$ fractional abundance are close to those found in the IGM prior to the onset of Pop. III star formation $\left(x_{\mathrm{e}, 0}=2 \times 10^{-4}, x_{\mathrm{H}_{2}, 0}=2 \times 10^{-6}\right)$. We assume that the gas is initially in charge balance, so that $x_{\mathrm{H}^{+}, 0}=x_{\mathrm{e}, 0}$, and place all of the hydrogen not accounted for by $\mathrm{H}^{+}$or $\mathrm{H}_{2}$ in atomic form. We also assume that the helium starts entirely in neutral atomic form, and that the initial abundances of the other three chemical species in our network $\left(\mathrm{H}^{-}, \mathrm{H}_{2}^{+}\right.$, and $\left.\mathrm{HeH}^{+}\right)$are zero. We note that these initial conditions are the same as those adopted in runs 2 and 5 in paper I.

\section{IMPACT OF CHEMICAL RATE COEFFICIENT UNCERTAINTIES ON $J_{\text {CRIT }}$}

\subsection{How sensitive is $J_{\text {crit }}$ to the rate of each reaction?}

As the starting point for our study, we first explore how sensitively our derived value of $J_{\text {crit }}$ depends on the rate coefficient of each of the reactions in our chemical model. We do this for purely pragmatical reasons: if the value of $J_{\text {crit }}$ is insensitive to the value of a particular rate coefficient, there is little point in spending time and effort critically assessing how well we know that value of that rate coefficient.

To assess how sensitive $J_{\text {crit }}$ is to variations in the rate coefficients of the reactions in our reduced network, we construct 52 different variants of our chemical model. In each of these variant models, the rate of one of the rate coefficients for our reduced set of 26 reactions is adjusted either upwards or downwards by a factor of $10^{0.5}$ from its fiducial value. We then determine $J_{\text {crit }}$ for each of these models, for both a T4 and a T5 spectrum. By comparing the outcome of the case in which a rate coefficient was adjusted upwards with the outcome of the case in which the same rate coefficient was adjusted downwards, we can quantify the effect of an order of magnitude change in that rate coefficient.

The results of this analysis are shown in Table 1] The sensitivity values we quote in the table are defined as the ratio of the largest and the smallest values of $J_{\text {crit }}$ that we obtain when modifying the rate coefficient of the specified reaction, i.e.

Sensitivity $=\frac{J_{\text {crit, } \max }}{J_{\text {crit, } \min }}$

With this definition, in a case where $J_{\text {crit }}$ depends linearly on the rate coefficient in question we would expect to obtain a sensitivity of 10 , while a value of 1 shows us that $J_{\text {crit }}$ is sensitive only to the inclusion of that reaction in the network, and not to the value of the rate coefficient adopted for the reaction.

From Table 1, we see that $J_{\text {crit }}$ is highly sensitive to the values of the rate coefficients for only a small subset of the reactions included in our minimal model. Specifically, if we restrict our attention to those reactions for which the sensitivity exceeds 1.25 (i.e. where an order of magnitude change in the rate coefficient results in a change in $J_{\text {crit }}$ of greater than $25 \%$ ), then we find that in runs performed with a T4 spectrum, the high sensitivity reactions are numbers 1-3, 58,10 and 12. In runs performed with a T5 spectrum, we find high sensitivities for a slightly different subset of reactions, numbers $1-3,5,6,8,10-12$, and 22 . This difference in behaviour is a consequence of the difference in the identity of the key process suppressing $\mathrm{H}_{2}$ cooling in the runs with different incident spectra. In runs with a $\mathrm{T} 4$ spectrum, $\mathrm{H}^{-}$ photodetachment plays a much greater role than $\mathrm{H}_{2}$ photodissociation in suppressing the $\mathrm{H}_{2}$ abundance. Therefore, we find that in these runs, $J_{\text {crit }}$ is only weakly sensitive to the $\mathrm{H}_{2}$ photodissociation rate (reaction 1), but has a much greater sensitivity to the $\mathrm{H}^{-}$photodetachment rate (reaction 7). On the other hand, in runs with a $\mathrm{T} 5$ spectrum, $\mathrm{H}_{2}$ photodissociation dominates, and so the value of $J_{\text {crit }}$ depends strongly on the rate of reaction 1 , but has hardly any sensitivity at all to the rate of reaction 7 , since in this case other reactions dominate the destruction of $\mathrm{H}^{-}$.

\subsection{Assessing uncertainties in the reaction rates}

In the previous section, we showed that $J_{\text {crit }}$ is highly sensitive to the values of the rates of only a small number of the reactions included in our reduced chemical model. In this subsection, we discuss how well we know the rates of these reactions. We restrict our attention to those reactions which have sensitivities greater than 1.25 for either the $\mathrm{T} 4$ or the T5 spectrum (or both).

\subsubsection{Photodissociation of $\mathrm{H}_{2}$ (reaction 1)}

The molecular data on which the calculation of the $\mathrm{H}_{2}$ photodissociation rate depends is known accurately (see e.g. Abgrall \& Roueff 1989), with an error of no more than a few percent. Therefore, in principle it should be possible to compute the $\mathrm{H}_{2}$ photodissociation rate produced by e.g. a T4 or T5 spectrum to a similar level of accuracy. However, in practice it is likely that the $\mathrm{H}_{2}$ photodissociation rates used in studies of the direct collapse model are rather less 
Table 1. Sensitivity of $J_{\text {crit }}$ to the rate coefficient adopted for each reaction in the reduced network

\begin{tabular}{|c|c|c|c|}
\hline No. & Reaction & Sensitivity (T4) & Sensitivity (T5) \\
\hline 1 & $\mathrm{H}_{2}+\gamma \rightarrow \mathrm{H}+\mathrm{H}$ & 1.66 & 9.52 \\
\hline 2 & $\mathrm{H}_{2}+\mathrm{H} \rightarrow \mathrm{H}+\mathrm{H}+\mathrm{H}$ & 16.0 & 9.21 \\
\hline 3 & $\mathrm{H}^{-}+\mathrm{H} \rightarrow \mathrm{H}_{2}+\mathrm{e}^{-}$ & 6.31 & 6.59 \\
\hline 4 & $\mathrm{H}_{2}^{+}+\mathrm{H} \rightarrow \mathrm{H}_{2}+\mathrm{H}^{+}$ & 1.10 & 1.02 \\
\hline 5 & $\mathrm{H}^{+}+\mathrm{e}^{-} \rightarrow \mathrm{H}+\gamma$ & 15.3 & 18.1 \\
\hline 6 & $\mathrm{H}+\mathrm{e}^{-} \rightarrow \mathrm{H}^{-}+\gamma$ & 7.01 & 33.3 \\
\hline 7 & $\mathrm{H}^{-}+\gamma \rightarrow \mathrm{H}+\mathrm{e}^{-}$ & 5.54 & 1.04 \\
\hline 8 & $\mathrm{H}+\mathrm{H}^{+} \rightarrow \mathrm{H}_{2}^{+}+\gamma$ & 3.89 & 1.40 \\
\hline 9 & $\mathrm{H}_{2}^{+}+\gamma \rightarrow \mathrm{H}^{+}+\mathrm{H}$ & 1.09 & 1.00 \\
\hline 10 & $\mathrm{H}+\mathrm{H} \rightarrow \mathrm{H}^{+}+\mathrm{e}^{-}+\mathrm{H}$ & 1.34 & 1.49 \\
\hline 11 & $\mathrm{H}^{-}+\mathrm{H} \rightarrow \mathrm{H}+\mathrm{H}+\mathrm{e}^{-}$ & 1.12 & 3.98 \\
\hline 12 & $\mathrm{H}+\mathrm{e}^{-} \rightarrow \mathrm{H}^{+}+\mathrm{e}^{-}+\mathrm{e}^{-}$ & 1.76 & 1.67 \\
\hline 13 & $\mathrm{H}_{2}^{+}+\mathrm{He} \rightarrow \mathrm{HeH}^{+}+\mathrm{H}$ & 1.00 & 1.00 \\
\hline 14 & $\mathrm{H}+\mathrm{He} \rightarrow \mathrm{H}^{+}+\mathrm{e}^{-}+\mathrm{He}$ & 1.10 & 1.14 \\
\hline 15 & $\mathrm{H}_{2}+\mathrm{H}^{+} \rightarrow \mathrm{H}_{2}^{+}+\mathrm{H}$ & 1.00 & 1.00 \\
\hline 16 & $\mathrm{H}_{2}+\mathrm{He} \rightarrow \mathrm{H}+\mathrm{H}+\mathrm{He}$ & 1.00 & 1.00 \\
\hline 17 & $\mathrm{HeH}^{+}+\mathrm{H} \rightarrow \mathrm{H}_{2}^{+}+\mathrm{He}$ & 1.00 & 1.00 \\
\hline 18 & $\mathrm{H}+\mathrm{H}+\mathrm{H} \rightarrow \mathrm{H}_{2}+\mathrm{H}$ & 1.00 & 1.00 \\
\hline 19 & $\mathrm{H}^{-}+\mathrm{He} \rightarrow \mathrm{H}+\mathrm{He}+\mathrm{e}^{-}$ & 1.00 & 1.02 \\
\hline 20 & $\mathrm{H}_{2}^{+}+\mathrm{H} \rightarrow \mathrm{H}+\mathrm{H}^{+}+\mathrm{H}$ & 1.00 & 1.00 \\
\hline 21 & $\mathrm{He}+\mathrm{H}^{+} \rightarrow \mathrm{HeH}^{+}+\gamma$ & 1.00 & 1.00 \\
\hline 22 & $\mathrm{H}^{-}+\mathrm{H}^{+} \rightarrow \mathrm{H}+\mathrm{H}$ & 1.05 & 1.75 \\
\hline 23 & $\mathrm{H}_{2}^{+}+\mathrm{e}^{-} \rightarrow \mathrm{H}+\mathrm{H}$ & 1.01 & 1.02 \\
\hline 24 & $\mathrm{HeH}^{+}+\mathrm{e}^{-} \rightarrow \mathrm{He}+\mathrm{H}$ & 1.00 & 1.00 \\
\hline 25 & $\mathrm{H}^{-}+\mathrm{H}^{+} \rightarrow \mathrm{H}_{2}^{+}+\mathrm{e}^{-}$ & 1.00 & 1.00 \\
\hline 26 & $\mathrm{H}^{-}+\mathrm{e}^{-} \rightarrow \mathrm{H}+\mathrm{e}^{-}+\mathrm{e}^{-}$ & 1.00 & 1.00 \\
\hline
\end{tabular}

The sensitivity quantifies the change in $J_{\text {crit }}$ that occurs when we change the value of the rate coefficient by a factor of ten.

accurate than this, owing to the approximations made when deriving them.

One potential source of error comes from the value adopted for the $\mathrm{H}_{2}$ photodissociation rate in optically thin gas (i.e. the rate in the absence of self-shielding). To compute this, it is necessary to specify the level populations of our collection of $\mathrm{H}_{2}$ molecules. Most studies of $\mathrm{H}_{2}$ photodissociation in primordial gas assume that the gas is at low density and hence that the effects of rotational and vibrational excitation can be ignored. In that case, the only energy levels of $\mathrm{H}_{2}$ that are populated are the $J=0$ para-hydrogen ground state and the $J=1$ ortho-hydrogen ground state. Some calculations of the $\mathrm{H}_{2}$ photodissociation rate further assume that only the $J=0$ state is populated (e.g. Abel et al. 1997), but in practice, if only the lowest rotational levels are populated, then the $\mathrm{H}_{2}$ photodissociation rate is insensitive to the ortho-to-para hydrogen ratio.

In dense gas, however, it is no longer reasonable to assume that only the $J=0$ and $J=1$ levels are populated. Instead, the populations of the excited rotational and vibrational levels will approach their LTE values. In LTE, the $\mathrm{H}_{2}$ photodissociation rate increases as the gas temperature increases, owing to the increasing importance of photodissociation from levels with $v>0$. This is illustrated in Figure 1 where we plot the $\mathrm{H}_{2}$ photodissociation rate in the LTE limit as a function of temperature, assuming either a T5 spectrum (solid line) or a T4 spectrum (dashed line). We see that in the case of a T5 spectrum, the photodissociation rate of hot, thermally populated $\mathrm{H}_{2}$ is around $30 \%$ larger than the rate at low temperatures, which in this case is the same as in the low density limit. In the case of the T4 spectrum,

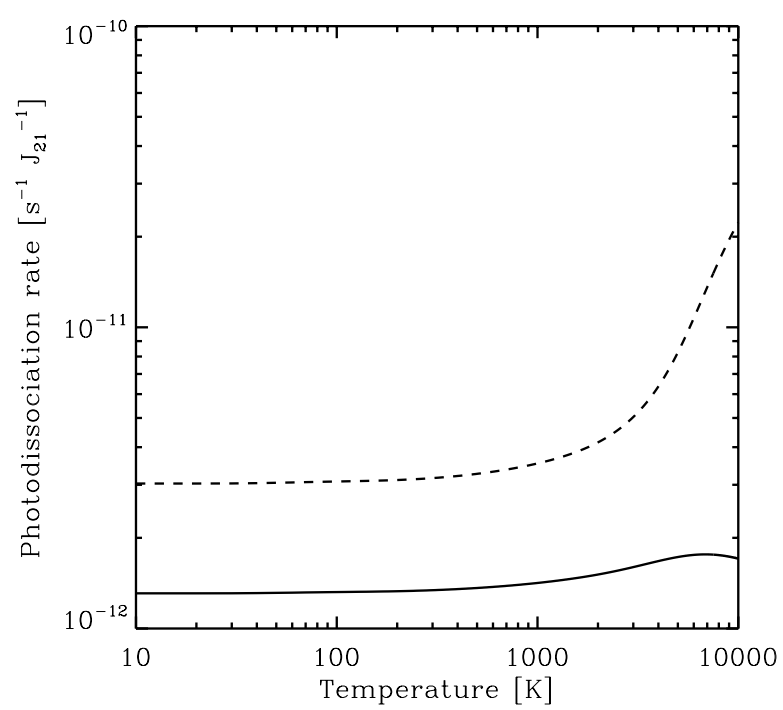

Figure 1. Temperature dependence of the $\mathrm{H}_{2}$ photodissociation rate in the LTE limit. Values are shown for both a T4 spectrum (dashed line) and a T5 spectrum (solid line). In both cases, we assume that $J_{21}=1$.

the temperature dependence of the rate is much stronger: it increases by roughly a factor of seven as we increase the temperature from $10 \mathrm{~K}$ to $10^{4} \mathrm{~K}$.

In order to help us assess how much influence the temperature dependence of the optically thin $\mathrm{H}_{2}$ photodissociation rate is likely to have on $J_{\text {crit }}$, we have examined the extreme case in which we adopt the LTE photodissociation 
rate throughout the collapse of the gas. We find that in this case, $J_{\text {crit }}$ is reduced to 12.3 if we use a T4 spectrum or 1210 if we adopt a T5 spectrum. Therefore, even in this extreme case, $J_{\text {crit }}$ changes by no more than around $30 \%$. In practice, the error introduced into $J_{\text {crit }}$ by the use of the low density limit for the optically thin $\mathrm{H}_{2}$ photodissociation rate will probably be smaller than this. This is because it is the behaviour of the gas at densities $n \sim 1000 \mathrm{~cm}^{-3}$ that determines the value of $J_{\text {crit }}$, and as this is less than the $\mathrm{H}_{2}$ critical density, $n_{\text {crit }} \sim 10^{4} \mathrm{~cm}^{-3}$, the $\mathrm{H}_{2}$ level populations will not yet have reached their LTE values.

A second effect that can potentially become important when the photodissociation rate is extremely strong is the photodissociation of $\mathrm{H}_{2}$ from highly excited vibrational levels. These levels can be populated by UV pumping of ground state $\mathrm{H}_{2}$ (Shull 1978, Draine \& Bertoldi 1996) or by so-called formation pumping, i.e. the tendency for newlyformed $\mathrm{H}_{2}$ to be in a highly excited rotational and vibrational state (see e.g. Launay et al. 1991). The typical lifetimes of highly excited vibrational states of $\mathrm{H}_{2}$ are short, of the order of $10^{6} \mathrm{~s}$ (Wolniewicz, Simbotin \& Dalgarno 1998), and so photodissociation of $\mathrm{H}_{2}$ from these states is significant only when the photodissociation rate is extremely large. This issue was investigated by Shull (1978), who showed that UV excitation of these highly excited vibrational levels becomes significant only for Lyman-Werner photon fluxes $F>3 \times 10^{-4}$ photons $\mathrm{cm}^{-2} \mathrm{~s}^{-1} \mathrm{~Hz}^{-1}$. If we convert this constraint to a constraint on $J_{21}$, we find that for a T5 spectrum it becomes approximately $J_{21}>5 \times 10^{5}$, while for a T4 spectrum we have $J_{21}>10^{5}$.

These values of $J_{21}$ are much larger than the values of $J_{\text {crit }}$ derived using our one-zone model. However, one-zone calculations tend to underestimate $J_{\text {crit }}$ compared to full $3 \mathrm{D}$ calculations, because they are unable to capture the complex temperature and density structure of the gas in real atomic cooling halos (see e.g. the comparison of one-zone and 3D results in Shang, Bryan \& Haiman 2010). 3D simulations performed using a T5 spectrum typically find that $J_{\text {crit }} \sim$ a few $\times 10^{4}$ (Shang, Bryan \& Haiman 2010 Latif et al. 2015), and models that account for the positive feedback from soft X-rays also find similarly high values (Inayoshi \& Tanaka 2015). At these values of $J_{21}$, radiative de-excitation of highly excited $\mathrm{H}_{2}$ molecules remains more effective than UV excitation, but the latter effect could potentially contribute at around the $10 \%$ level. One effect of this will be to slightly suppress the $\mathrm{H}_{2}$ formation rate, as some fraction of the newly formed, vibrationally hot $\mathrm{H}_{2}$ molecules will be photodissociated before they can radiatively decay to the vibrational ground state. Another effect will be to slightly enhance the total $\mathrm{H}_{2}$ photodissociation rate (Shull 1978). A detailed accounting for the effect of this on the final value of $J_{\text {crit }}$ is far outside the scope of the present work, but it seems likely that the effect will be of the order of $10 \%$ or less.

Lastly, but most importantly, the values of $J_{\text {crit }}$ that we derive in simulations performed using a $\mathrm{T} 5$ spectrum are highly sensitive to the treatment of $\mathrm{H}_{2}$ self-shielding adopted in the simulation (Wolcott-Green, Haiman \& Bryan 2011. Sugimura, Omukai \& Inoue 2014). For ease of comparison to other recent calculations, the simulations presented in this paper were performed using the modified version of the Draine \& Bertoldi (1996) $\mathrm{H}_{2}$ self-shielding function given in Wolcott-Green, Haiman \& Bryan (2011). This version of the self-shielding function is intended for use when one has rotationally hot $\mathrm{H}_{2}$, i.e. when the $\mathrm{H}_{2}$ rotational level populations are in LTE (although Wolcott-Green, Haiman \& Bryan 2011 assume that the vibrational level populations continue to be negligible). On the other hand, the original Draine \& Bertoldi (1996) self-shielding function,

$$
\begin{aligned}
f_{\text {sh }, \text { orig }}\left(N_{\mathrm{H}_{2}}, T\right)= & \frac{0.965}{\left(1+x / b_{5}\right)^{2.0}}+\frac{0.035}{(1+x)^{0.5}} \\
& \times \exp \left[-8.5 \times 10^{-4}(1+x)^{0.5}\right],
\end{aligned}
$$

with $x=N_{\mathrm{H}_{2}} / 5 \times 10^{14} \mathrm{~cm}^{-2}$ and $b_{5}=b / 10^{5} \mathrm{~cm} \mathrm{~s}^{-1}$ gives a better fit when the $\mathrm{H}_{2}$ is rotationally cold, with significant populations only in the $J=0$ and $J=1$ rotational levels. Depending on which version of $f_{\mathrm{sh}}$ one chooses, one recovers very different values for $J_{\text {crit }}$ in runs with a T5 spectrum (Sugimura, Omukai \& Inoue 2014). For example, in our model, using $f_{\text {sh, orig }}$ in place of $f_{\text {sh }}$ increases $J_{\text {crit }}$ from 1640 to approximately 9000 .

This prompts the question of which version of the selfshielding function one should adopt for these calculations. Unfortunately, this is not an easy question to answer. As we have already discussed, at the density at which $J_{\text {crit }}$ is determined in these simple one-zone calculations, the $\mathrm{H}_{2}$ level populations have not yet reached LTE. Nevertheless, the density is high enough that collisional population of levels with $J>1$ is starting to become important, and so we cannot simply assume that the $\mathrm{H}_{2}$ molecules are rotationally cold. It is likely that reality lies somewhere between these two limiting cases, but how far in between is difficult to say. Clarifying this will require one to track the evolution of the $\mathrm{H}_{2}$ rotational level populations during the collapse, a task which lies outside of the scope of this present study.

Finally, in addition to this sensitivity to the choice of $\mathrm{H}_{2}$ self-shielding function, the value of $J_{\text {crit }}$ is also sensitive to the method used to determine $N_{\mathrm{H}_{2}}$. Most one zone calculations adopt the simple approximation $N_{\mathrm{H}_{2}}=n_{\mathrm{H}_{2}} L_{\mathrm{char}}$, where $L_{\text {char }}$ is some characteristic length scale, but different authors define this different length scale in different ways. There is general agreement that it should be related to the Jeans length, but no consensus on whether $L_{\text {char }}=\lambda_{\mathrm{J}}$ or $L_{\text {char }}=\lambda_{\mathrm{J}} / 2$. Moreover, it is not always entirely clear what definition is being used for $\lambda_{\mathrm{J}}$ - different definitions in the literature can easily disagree by a factor of two, depending on whether one derives $\lambda_{\mathrm{J}}$ from the dispersion relation for a $1 \mathrm{D}$ plane wave or by considering the balance between thermal and gravitational energy in a uniform density spherical cloud. Of course, in reality none of these prescriptions is correct - Wolcott-Green, Haiman \& Bryan (2011) have shown that the Jeans length approximation can yield values of $N_{\mathrm{H}_{2}}$ and $f_{\mathrm{sh}}$ that differ by an order of magnitude or more from the true values that one derives by post-processing $3 \mathrm{D}$ simulations. To eliminate this source of error, it is necessary to abandon the use of simple approximations for $N_{\mathrm{H}_{2}}$, and to compute it self-consistently within a simulation using an algorithm such as TreeCol (Clark, Glover \& Klessen 2012). An attempt to do just this is reported on by Hartwig et al. (2015), but their results lie outside of the scope of the present study. 


\subsubsection{Collisional dissociation of $\mathrm{H}_{2}$ by $\mathrm{H}$ (reaction 2)}

As discussed in e.g. Martin, Schwarz \& Mandy (1996), there are two separate processes that contribute to the rate of this reaction. First, there is direct collisional dissociation, involving a transition from a bound state of the $\mathrm{H}_{2}$ molecule directly into the continuum of classically unbound states. Second, there is dissociative tunneling, which involves the excitation of the $\mathrm{H}_{2}$ molecule into a quasi-bound state - a state that has an internal energy larger than is required for dissociation, but which is separated from the continuum by a barrier in the effective potential. $\mathrm{H}_{2}$ molecules in these highly excited quasi-bound states dissociate only if they can tunnel through the barrier before they have a chance to deexcite to a fully bound state. At temperatures $T<4500 \mathrm{~K}$, this second process dominates the total collisional dissociation rate (Martin, Keogh \& Mandy 1998), and it remains important even at significantly higher temperatures. Some previous studies of the direct collapse model have accounted only for the direct collisional dissociation of $\mathrm{H}_{2}$, and not for the influence of dissociative tunneling (see e.g. Shang, Bryan \& Haiman 2010). In paper I, we showed that the neglect of dissociative tunnelling can lead to one overestimating $J_{\text {crit }}$ by up to a factor of two; similar results have also been reported recently by Latif et al. (2014).

Even if one does account for both processes, uncertainties in the rate coefficients adopted will also introduce some uncertainty into $J_{\text {crit }}$. In our simulations, we adopt rate coefficients for these processes based on the master equation study of Martin, Schwarz \& Mandy (1996). They present complicated fitting functions for both rate coefficients that account for their dependence on both the temperature and the density of the gas. The Martin et al. study itself relies on state-to-state collisional rate coefficients and collisional dissociation rates for $\mathrm{H}-\mathrm{H}_{2}$ collisions computed by Mandy \& Martin (1993) using the quasi-classical trajectories (QCT) method and the LSTH potential energy surface (Liu 1973 Truhlar \& Horowitz 1978; Siegbahn \& Liu 1978).

The use of the QCT method instead of a fully quantal method introduces some inaccuracy into the rate coefficients. At low temperatures, very large differences are found between the two methods (see e.g. Sun \& Dalgarno 1994. Boothroyd et al. 1996), but much better agreement is found at high temperatures, and so the QCT results should be reasonably reliable at the temperatures relevant for $\mathrm{H}_{2}$ collisional dissociation. Similarly, differences between the LSTH potential energy surface (PES) and more recent parameterizations of the $\mathrm{H}_{3}$ PES (e.g. Varandas et al. 1987||Boothroyd et al. 1991, 1996, Mielke, Garrett \& Peterson 2002) are important at low temperatures (compare, e.g., the results presented in Sun \& Dalgarno 1994. Lepp, Buch \& Dalgarno 1995 and Wrathmall \& Flower 2007), particularly for pure rotational transitions, but appear to become much less important at high temperatures (Martin, Keogh \& Mandy 1998). In the absence of a comprehensive treatment of $\mathrm{H}_{2}$ excitation by $\mathrm{H}$ along the lines of the Martin et al. study but using collisional rates computed with a different PES, it is difficult to state with certainty the total error introduced into the collisional dissociation rate by the use of an older version of the PES. However, the comparison between dissociation rate coefficients computed using the LSTH surface and the BKMP2 surface of Boothroyd et al. (1996) shown in Figure 2 of Esposito \& Capitelli (2009) suggests that the error must be relatively small at the temperatures of interest in this study. A conservative estimate for the error would be $25 \%$. This introduces an error into $J_{\text {crit }}$ of roughly $35 \%$ in simulations with a T4 spectrum and $25 \%$ in simulations with a T5 spectrum.

\subsubsection{Associative detachment of $H^{-}$with $H$ (reaction 3)}

The rate of this reaction has recently been measured by Kreckel et al. (2010) for temperatures in the range $1<$ $T<10^{4} \mathrm{~K}$. The systematic error in these measurements is approximately $25 \%$. The results agree well with the low temperature measurements of Gerlich et al. (2012) and the calculations of Cízek et al. (1998), differing by amounts that are less than this systematic error. We have investigated the effect of this uncertainty in the rate of reaction 3 by scaling the rate coefficient both up and down by $25 \%$ and determining what effect this has on our estimate of $J_{\text {crit }}$. In runs with a T4 spectrum, we find that $J_{\text {crit }}=15.1$ when we decrease the rate coefficient by $25 \%$ and $J_{\text {crit }}=21.6$ when we increase the rate coefficient by $25 \%$. In runs with a $\mathrm{T} 5$ spectrum, the corresponding values are $J_{\text {crit }}=1350$ and 1930 . The remaining uncertainty in the rate of reaction 3 therefore introduces an uncertainty of around $40 \%$ into our estimates of $J_{\text {crit }}$.

\subsubsection{Radiative recombination of $\mathrm{H}^{+}$(reaction 5)}

The rate of this reaction is known extremely accurately and high quality analytical fits are available that describe the temperature dependence of the rate coefficient. For example, the fit that we use in our study for the case B recombination rate coefficient - taken from Hui \& Gnedin (1997) and based on the data presented in Ferland et al. (1992) - has a quoted fitting error of only $0.7 \%$ over the temperature range $1<$ $T<10^{9} \mathrm{~K}$. Therefore, although $J_{\text {crit }}$ is highly sensitive to the rate of this reaction, the high accuracy with which the rate is known means that in general, this reaction does not contribute significantly to the uncertainty in our estimates of $J_{\text {crit }}$.

That said, one must still take care to use the correct recombination rate coefficient. Gravitationally collapsing gas within an atomic cooling halo generally has a low fractional ionization, and hence the mean free path for ionizing photons is small. For this reason, the on-the-spot approximation generally applies, and when modelling the recombination of $\mathrm{H}^{+}$one should therefore use the case $\mathrm{B}$ recombination rate coefficient. If the case A rate coefficient is used instead (as in e.g. the original Abel et al. 1997 chemical model for primordial gas, or the recent simulations by Latif et al. 2015), and the ionizing photons produced by direct recombination to the atomic hydrogen ground state are not explicitly accounted for, then the net effect is to increase the recombination rate by roughly $60 \%$ in the temperature range of interest. To assess the effect that this would have on $J_{\text {crit }}$, we have re-run our simulations, using the case A recombination rate coefficient from Hui \& Gnedin (1997) in place of the case $\mathrm{B}$ value. With this change, we find that $J_{\text {crit }}=10.1$ for a T4 spectrum and $J_{\text {crit }}=871$ for a T5 spectrum, demonstrating that the use of the wrong rate coefficient introduces an $80-90 \%$ error into our estimate of $J_{\text {crit }}$. 


\subsubsection{Radiative association of $H$ and $e^{-}$(reaction 6)}

Calculations of this rate in the literature generally derive it by applying detailed balance to the inverse process, $\mathrm{H}^{-}$ photodissociation (reaction 7 ). Since the $\mathrm{H}^{-}$photodissociation cross-section is known accurately, one would expect that there should be little uncertainty in the resulting rate coefficient. However, when constructing an analytical fit to the calculated rate coefficient, it is necessary to choose some range of temperatures over which to fit. If the results of these fits are then extrapolated outside of their range of validity, then there is no guarantee that the resulting rate coefficients will remain accurate, and in this case, different fits may then yield very different results. This effect is seen quite clearly in Figure 2, where we compare several different analytical fits given in the literature. We plot results using the fits given in Hutchins (1976) (dotted line), Abel et al. (1997) (dashed line) and Stancil, Lepp \& Dalgarno (1998) (dot-dashed line), in each case showing the ratio of the values given by these fits to those given by the fit in Galli \& Palla (1998).

We see from Figure 2 that all four prescriptions agree well for gas temperatures in the range $10<T<2500 \mathrm{~K}$. Within this temperature range, typical differences between different versions of the rate coefficient are of the order of $10 \%$ or less. At temperatures $T>2500 \mathrm{~K}$, however, the Hutchins (1976) fit starts to diverge strongly from the other rates. This behaviour is unsurprising, as the range of validity quoted by Hutchins for the fit is $100<T<2500 \mathrm{~K}$.

Similarly, at $T>6000 \mathrm{~K}$, the other three versions of the rate coefficient start to differ significantly: the Abel et al. (1997) version increases more strongly with increasing temperature than the Galli \& Palla (1998) version, while the Stancil, Lepp \& Dalgarno (1998) version of the rate coefficient increases less strongly than the Galli \& Palla (1998) version. This difference in behaviour is presumably due to some or all of the analytical fits reaching the end of their temperature range of validity at $T \sim 6000 \mathrm{~K}$, although it is difficult to be certain as none of the other papers state the temperature range over which their fits are supposed to be valid.

We have investigated the effect that this difference in rates has on the value of $J_{\text {crit }}$. For runs with a T4 spectrum, we find that $J_{\text {crit }}=16.9,18.1,20.0$ and 25.4 when we use the rate coefficient from Stancil, Lepp \& Dalgarno (1998), Abel et al. (1997), Galli \& Palla (1998) or Hutchins (1976), repsectively. For runs with a $\mathrm{T} 5$ spectrum, the corresponding values are $J_{\text {crit }}=1500,1630,1970$ and 2840 .

We know from our own work in Paper I and from the work of previous authors (e.g. Shang, Bryan \& Haiman 2010) that the value of $J_{\text {crit }}$ is primarily determined by the behaviour of the $\mathrm{H}_{2}$ fraction in gas with a density $n \sim 10^{3} \mathrm{~cm}^{-3}$ and a temperature $T \sim 7500 \mathrm{~K}$. In these conditions, the rate coefficient for reaction 6 given by Hutchins (1976) is not valid, and consequently it is no surprise that the value of $J_{\text {crit }}$ that we recover when we use this rate differs significantly from those recovered using any of the other analytical fits. We do not recommend use of the Hutchins (1976) rate in future studies of the direct collapse model.

As far as the other three fits are concerned, there seems to be no particular reason to prefer one over the others at the present time. We can therefore conclude that the current

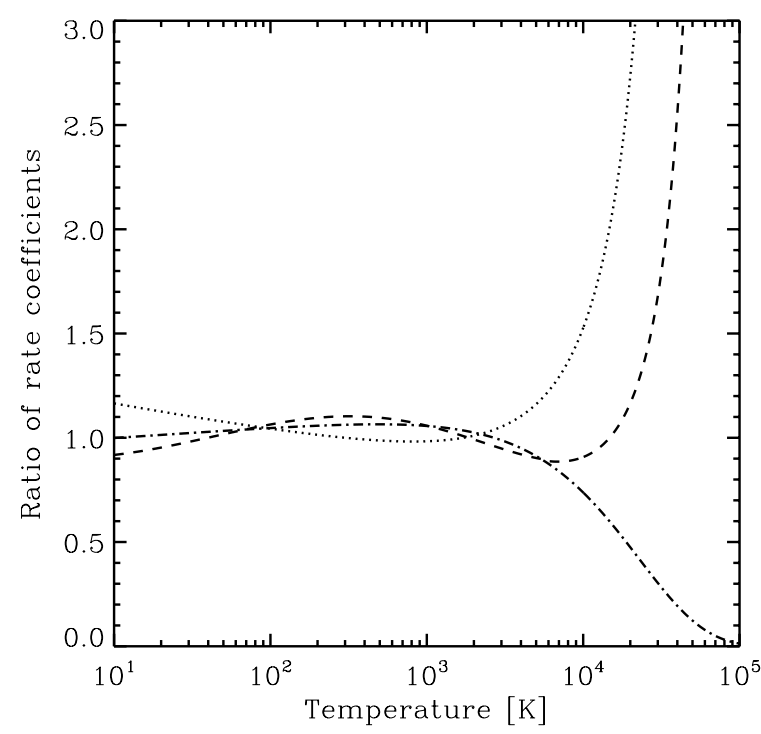

Figure 2. Comparison of different determinations of the rate coefficient for the radiative association of $\mathrm{H}$ and $\mathrm{e}^{-}$(reaction 6). We show results for three different analytical fits, taken from Hutchins (1976) (dotted line), Abel et al. (1997) (dashed line), and Stancil, Lepp \& Dalgarno (1998) (dot-dashed line). In each case, we plot the ratio of the rate coefficient given by the analytic fits presented in these papers with that presented in the review of Galli \& Palla (1998).

uncertainty in this rate coefficient introduces an uncertainty of between $20-30 \%$ into the value of $J_{\text {crit }}$.

Finally, it should be noted that the uncertainty in this rate coefficient may also affect the later thermal evolution of the gas in halos where $J_{21}>J_{\text {crit }}$, as in this case, $\mathrm{H}^{-}$boundfree emission becomes one of the most important cooling processes once the density exceeds $n \sim 10^{8} \mathrm{~cm}^{-3}$ (see e.g. Omukai 2001. Schleicher, Spaans \& Glover 2010). However, a detailed study of this issue is outside of the scope of the present paper.

\subsubsection{Photodissociation of $H^{-}$(reaction 7)}

Accurate cross-sections for this process are given by de Jong (1972) and Wishart (1979). More recently, Miyake et al. (2010) have computed revised cross-sections that account for the effect of the prominent resonances in the cross-section at photon energies $h \nu>11 \mathrm{eV}$. However, the effect of these resonances on the photodissociation rate is small: for a $\mathrm{T} 4$ spectrum, the rate is enhanced by no more than a couple of percent, while for a T5 spectrum, the enhancement is around $20 \%$. We have investigated the effect of this enhancement on $J_{\text {crit }}$, and find that for the T4 spectrum, it is reduced by a few percent, to $J_{\text {crit }}=17.7$, while for the T5 spectrum, it is not significantly affected. It should also be noted that this small chemical uncertainty is dwarfed by the much larger astrophysical uncertainty arising from the fact that both the T4 and T5 spectra are relatively crude approximations to the spectra of real high-redshift protogalaxies (Sugimura, Omukai \& Inoue 2014, Agarwal \& Khochfar 2015, Latif et al. 2015 Agarwal et al. 2015). 


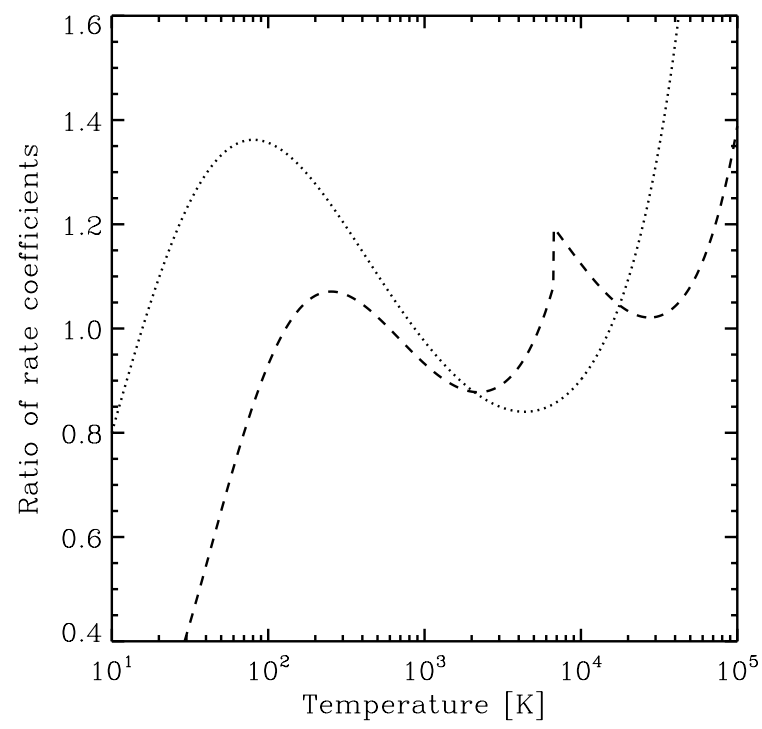

Figure 3. Comparison of different analytical fits for the rate coefficient for the radiative association of $\mathrm{H}$ and $\mathrm{H}^{+}$(reaction 8). The dotted line shows the ratio of the fit given in Galli \& Palla (1998) to that in Latif et al. (2015), while the dashed line shows the ratio of the Shapiro \& Kang (1987) fit to the Latif et al. (2015) fit. Comparison of the Latif et al. fit with the rates tabulated in Ramaker \& Peek (1976) shows that it agrees to within a few percent at the temperatures of interest.

\subsubsection{Radiative association of $H$ and $H^{+}$(reaction 8)}

Rate coefficients for this reaction have been computed by both Ramaker \& Peek (1976) and Stancil, Babb \& Dalgarno (1993), and agree to within 3\%. Unfortunately, there is not such a good level of agreement between the different fits to the Ramaker \& Peek (1976) results that have been used in the literature. Shapiro \& Kang (1987) introduced a fit of the form

$k_{8, \mathrm{SK}}=1.85 \times 10^{-23} T^{1.8} \mathrm{~cm}^{3} \mathrm{~s}^{-1}$

for $T<6700 \mathrm{~K}$ and

$k_{8, \mathrm{SK}}=5.81 \times 10^{-16}\left(\frac{T}{56200}\right)^{-0.6657 \log (T / 56200)} \mathrm{cm}^{3} \mathrm{~s}^{-1}(6)$

for $T>6700 \mathrm{~K}$ that was later adopted by many other authors; for instance, it is this prescription that is currently used in the ENZO primordial chemistry model, as used in e.g. Shang, Bryan \& Haiman (2010). However, in their review of primordial chemistry, Galli \& Palla (1998) use a different fit, ostensibly to the same data

$$
\begin{aligned}
k_{8, \mathrm{GP}}= & \operatorname{dex}\left[-19.38-1.523 \log T+1.118(\log T)^{2}\right. \\
& \left.-0.1269(\log T)^{3}\right] \mathrm{cm}^{3} \mathrm{~s}^{-1} .
\end{aligned}
$$

Finally, in a recent paper, Latif et al. (2015) give the fit

$$
\begin{aligned}
k_{8, \mathrm{~L}}= & \operatorname{dex}\left[-18.20-3.194 \log T+1.786(\log T)^{2}\right. \\
& \left.-0.2072(\log T)^{3}\right] \mathrm{cm}^{3} \mathrm{~s}^{-1},
\end{aligned}
$$

citing Coppola et al. (2011) as the source.

In Figure 3 we compare these three different versions of the rate coefficient by plotting the ratios $k_{8, \mathrm{SK}} / k_{8, \mathrm{~L}}$ and $k_{8, \mathrm{GP}} / k_{8, \mathrm{~L}}$ as a function of temperature. We see that at the temperatures of interest, the scatter in the values of the three fits is around $30 \%$, ten times larger than the difference between the Ramaker \& Peek (1976) and Stancil, Babb \& Dalgarno (1993) calculations. This scatter has an appreciable effect on the values of $J_{\text {crit }}$ that we recover for runs performed using a T4 spectrum. We find that for runs using the Shapiro \& Kang (1987), Galli \& Palla (1998) and Latif et al. $(2015)$ fits, $J_{\text {crit }}=19.8,16.8$, and 18.0, respectively, a total difference of around $20 \%$. A similar analysis carried out for runs using a T5 spectrum finds a much smaller range of values: for the same three cases, $J_{\text {crit }}=1670,1600$, and 1630 , giving us a total uncertainty in this case of only $4 \%$.

We have compared the values predicted by these three different versions of the rate coefficient with the values tabulated by Ramaker \& Peek (1976), and find that the Latif et al. (2015) fit gives the best overall match to the tabulated data, agreeing to within around $4-5 \%$ over the entire temperature range considered by Ramaker \& Peek $(10<T<32000 \mathrm{~K})$. We therefore recommend that this fit to the rate coefficient should be used in future numerical studies of the direct collapse model.

\subsubsection{Collisional ionization of $H$ by $H$ (reaction 10)}

Although there have been a number of calculations and measurements of the cross-section for this process at high collision energies (see e.g. McClure 1968, Hill, Geddes \& Gilbody 1979, Shingal, Bransden \& Flower 1989 Riley \& Burke 1999), only a few studies have looked at its behaviour at the low energies relevant for the direct collapse model. In our fiducial model, we use the rate coefficient for this reaction given in Lenzuni, Chernoff \& Salpeter (1991):

$k_{10, \mathrm{LCS}}=1.2 \times 10^{-17} T^{1.2} \exp \left(-\frac{157800}{T}\right) \mathrm{cm}^{3} \mathrm{~s}^{-1}$.

This is based on the experimental cross-sections of Gealy \& van Zyl (1987). However, their measurements only exist for collision energies $E>100 \mathrm{eV}$. Therefore, in order to derive a rate coefficient that is valid at temperatures $T \sim 10^{4} \mathrm{~K}$, it is necessary to extrapolate the measured cross-sections down to the ionization threshold energy of $13.6 \mathrm{eV}$. This extrapolation can potentially introduce a substantial error into the resulting rate coefficient. Indeed, a good example of this is provided by the fact that Hollenbach \& McKee (1989), using the same cross-section data, derive a rate coefficient

$k_{10, \mathrm{HM} 89}=1.7 \times 10^{-14} T^{0.5} \exp \left(-\frac{149000}{T}\right) \mathrm{cm}^{3} \mathrm{~s}^{-1}$.

Although based on the same experimental data, these two expressions differ by a factor of 5 at $T=10^{4} \mathrm{~K}$ and by an order of magnitude at $T=7000 \mathrm{~K}$.

The $\mathrm{H}-\mathrm{H}$ collisional ionization rate coefficient can also be derived using the semi-empirical method described in Drawin (1968, 1969). Following the interpretation of Drawin]'s results given in Soon (1992), we can write the rate coefficient as

$k_{10, \mathrm{D} 69}=1.57 \times 10^{-14} T^{0.5} \psi(x) \mathrm{cm}^{3} \mathrm{~s}^{-1}$,

where

$\psi(x)=\left(1+\frac{2}{x}\right)\left[\frac{1}{1+\left(2 m_{\mathrm{e}} / m_{\mathrm{H}} x\right)^{2}}\right] \exp (-x)$,

$m_{\mathrm{e}}$ is the electron mass, $m_{\mathrm{H}}$ is the mass of a hydrogen atom, and $x=157800 / T$. 
Yet another expression for the $\mathrm{H}-\mathrm{H}$ collisional ionization rate coefficient can be found in Hollenbach \& McKee (1979). They assume that it is a constant factor of $1.7 \times 10^{-4}$ smaller than the $\mathrm{H}_{-} \mathrm{e}^{-}$rate (citing Drawin 1969 in support of this fact), and hence write the rate coefficient as

$k_{10, \mathrm{HM} 79}=9.8 \times 10^{-15} T^{0.5} \exp \left(-\frac{157800}{T}\right) \mathrm{cm}^{3} \mathrm{~s}^{-1}$.

Finally, analytical expressions for the $\mathrm{H}-\mathrm{H}$ ionization cross-section and corresponding thermal rate coefficient are given in Soon (1992). These result from an extension of the work of Kunc \& Soon (1991), and were calculated using the classical impulse approximation (Gryzinski 1965). Unfortunately, there are a couple of significant problems with the expressions presented in Soon's paper, as already noted by Barklem (2007). Most importantly, Soon uses the wrong value for the ionization threshold energy. In the centre-ofmass frame, this should be equal to the ionization potential of hydrogen, $I_{\mathrm{H}}=13.6 \mathrm{eV}$. However, Soon adopts a threshold which is half of this value, for reasons which are unclear (see the lengthy discussion in Barklem 2007). In the high energy regime probed by experiments, this error is unimportant, but at the low temperatures that we are interested in, the choice of threshold has a huge effect on the rate coefficient, as we demonstrate below. In addition, Barklem reports that the rate coefficient that one obtains by numerically integrating the ionization cross-section given by Soon (using Soon's choice of threshold) differs significantly at $T<10^{5} \mathrm{~K}$ from the collisional ionization rate coefficient given by Soon.

In view of these issues, we have chosen to proceed by ignoring the expressions given in Soon (1992) and instead analytically integrating the cross-section given in Kunc \& Soon (1991), using the correct value for the energy threshold. At the low energies of interest here, this yields a rate

$k_{10, \mathrm{KS} 91}=4.65 \times 10^{-21} T^{3 / 2} \exp \left(-\frac{157800}{T}\right) \mathrm{cm}^{3} \mathrm{~s}^{-1}$.

We compare these five different expressions for the $\mathrm{H}-\mathrm{H}$ collisional ionization rate in Figure 4. We see that the rate derived from the Kunc \& Soon (1991) cross-section has an energy dependence that agrees well with the other versions of the rate coefficient. However, there is a significant disagreement when it comes to the normalization of the rate, with this value being around a factor of 100 smaller than the others at all temperatures. Fortunately, the impact of this uncertainty on $J_{\text {crit }}$ is relatively small. Using a small value for the rate coefficient of reaction 10 has a similar effect to omitting it entirely, which as we saw in Paper I leads to a decrease in $J_{\text {crit }}$ of around $20-30 \%$.

The remainder of the rate coefficients agree much better in terms of both their temperature dependence and their normalization. Even so, there remains a substantial scatter in the values at low temperatures. For example, as previously noted, the Hollenbach \& McKee (1989) and Lenzuni, Chernoff \& Salpeter (1991) rates differ by around an order of magnitude at $T=7000 \mathrm{~K}$, despite being based on the same atomic data. This scatter introduces a significant uncertainty into $J_{\text {crit }}$ : in runs with a T4 spectrum, the value of $J_{\text {crit }}$ varies by up to $80 \%$ depending on which version of the $\mathrm{H}-\mathrm{H}$ collisional ionization rate coefficient we adopt, while in runs with a T5 spectrum, the uncertainty is even larger, around a factor of 2.5

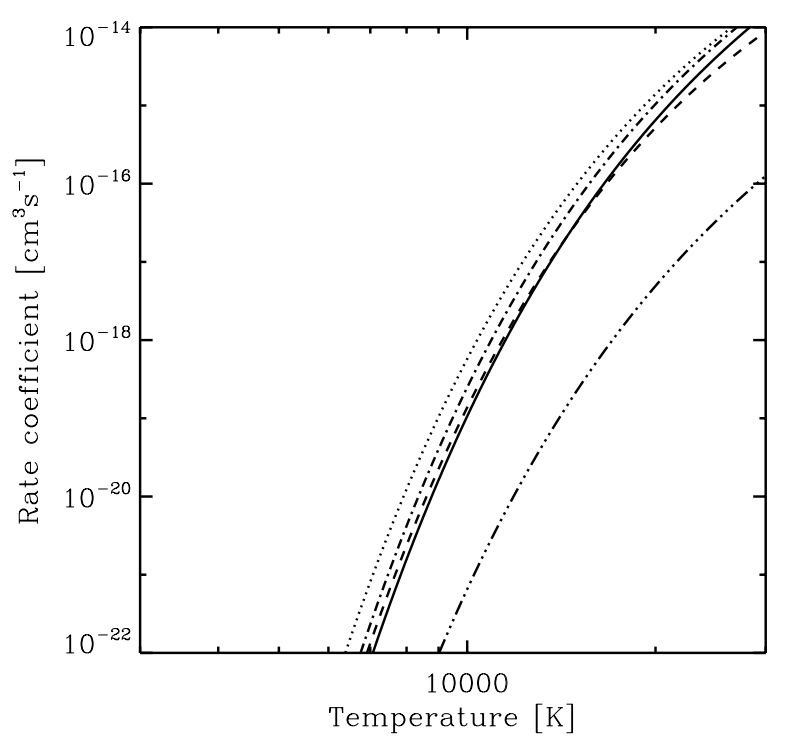

Figure 4. Comparison of different rate coefficients for the collisional ionization of $\mathrm{H}$ by $\mathrm{H}$ (reaction 10). The values shown are taken from Lenzuni, Chernoff \& Salpeter (1991) (solid line), Hollenbach \& McKee (1989) (dotted line), Hollenbach \& McKee (1979) (lower dashed line), Drawin (1969) (dash-dotted line), and this work, based on the cross-section of Kunc \& Soon (1991) (dotdot-dot-dashed line).

Finally, there remains the question of which of the different rate coefficients one should choose for future calculations. As the Kunc \& Soon (1991) cross-section agrees very well with the available experimental data at $E>100 \mathrm{eV}$ (see e.g. figure 3 in their paper), the rate coefficient based on this cross-section is probably the most accurate one available at the present time. However, the fact that there is a large gap between the range of energies for which the cross-section is experimentally constrained and the range of interest for deriving the low-temperature form of the rate coefficient means that we must treat any of the possible rate coefficients with considerable caution.

\subsubsection{Collisional detachment of $H^{-}$by $H$ (reaction 11)}

Considerable uncertainty remains in the rate of this reaction. An early study by Browne \& Dalgarno (1969) gives two different sets of values for the reaction rate, depending on what form is chosen for the interaction potential. If the Bardsley, Herzenberg \& Mandl (1966a b potential is adopted, then their numerical results are fit to within $10-20 \%$ by the expression

$k_{11, \mathrm{BD} 66}=1.74 \times 10^{-12} T^{0.79} \exp \left(\frac{-8760}{T}\right) \mathrm{cm}^{3} \mathrm{~s}^{-1}$.

On the other hand, if the Chen \& Peacher (1968) potential is used, we instead obtain a rate coefficient that is well fit by the expression

$k_{11, \mathrm{CP} 68}=1.74 \times 10^{-13} T^{0.94} \exp \left(\frac{-8760}{T}\right) \mathrm{cm}^{3} \mathrm{~s}^{-1}$.

More recently, cross-sections for this reaction were given in the reviews of Janev, Langer \& Evans (1987) and Janev, Reiter \& Samm (2003). Based on the Janev, Langer \& Evans 
(1987) data, Lenzuni, Chernoff \& Salpeter (1991) propose a rate coefficient of the form

$k_{11, \mathrm{LCS} 91}=4.3 \times 10^{-16} \mathrm{~T}^{1.58} \mathrm{~cm}^{3} \mathrm{~s}^{-1}$.

Abel et al. (1997) use the same cross-section data, but give a different analytical fit. At temperatures $T_{\mathrm{e}}<0.1$, where $T_{\mathrm{e}}=T / 11604.4 \mathrm{~K}$, their fit has the form

$k_{11, \mathrm{~A} 97}=2.5634 \times 10^{-9} T_{\mathrm{e}}^{1.78186} \mathrm{~cm}^{3} \mathrm{~s}^{-1}$,

while at higher temperatures, it has the form

$$
\begin{aligned}
k_{11, \mathrm{~A} 97}= & \exp [-20.37260896 \\
& +1.13944933\left(\ln T_{\mathrm{e}}\right) \\
& -0.14210135\left(\ln T_{\mathrm{e}}\right)^{2} \\
& +8.4644554 \times 10^{-3}\left(\ln T_{\mathrm{e}}\right)^{3} \\
& -1.4327641 \times 10^{-3}\left(\ln T_{\mathrm{e}}\right)^{4} \\
& +2.0122503 \times 10^{-4}\left(\ln T_{\mathrm{e}}\right)^{5} \\
& +8.6639632 \times 10^{-5}\left(\ln T_{\mathrm{e}}\right)^{6} \\
& -2.5850097 \times 10^{-5}\left(\ln T_{\mathrm{e}}\right)^{7} \\
& +2.4555012 \times 10^{-6}\left(\ln T_{\mathrm{e}}\right)^{8} \\
& \left.-8.0683825 \times 10^{-8}\left(\ln T_{\mathrm{e}}\right)^{9}\right] \mathrm{cm}^{3} \mathrm{~s}^{-1} .
\end{aligned}
$$

Finally, the more recent data from Janev, Reiter \& Samm (2003) can be used to derive a rate coefficient which is fit to within $1 \%$ over the temperature range $30<T<30000 \mathrm{~K}$ by the function

$$
\begin{aligned}
k_{11, \mathrm{~J} 03}= & \operatorname{dex}[-10.911243 \\
& +2.0648286\left(\log T_{3}\right) \\
& -0.40506856\left(\log T_{3}\right)^{2} \\
& +0.16462528\left(\log T_{3}\right)^{3} \\
& -0.10911689\left(\log T_{3}\right)^{4} \\
& \left.+0.022537886\left(\log T_{3}\right)^{5}\right] \mathrm{cm}^{3} \mathrm{~s}^{-1},
\end{aligned}
$$

where $T_{3}=T / 1000 \mathrm{~K}$.

In Figure 5 , we show how these different versions of the rate coefficient behave as a function of temperature. We see immediately that at temperatures below a few thousand $\mathrm{K}$, there is a large uncertainty in the value of the rate coefficient. The values from Browne \& Dalgarno (1969) fall off much more rapidly with decreasing temperature than the other versions of the rate coefficient. Moreover, even if we ignore this early work, and restrict our attention to the results of more recent studies, we see that there is still a large uncertainty in the low temperature behaviour, with the rate coefficient that we have derived from the data in Janev, Reiter \& Samm (2003) falling off more rapidly at low temperatures than the Lenzuni, Chernoff \& Salpeter (1991) or Abel et al. (1997) rates. Fortunately, the chemical evolution of the gas is unlikely to be sensitive to this large uncertainty. The reason for this is that at the temperatures where there is an order of magnitude or more disagreement between the different rate coefficients, all of the expressions listed here predict values that are much smaller than the associative detachment rate. Therefore, in this regime, associative detachment dominates regardless of which expression we adopt for the rate of reaction 11 .

Of greater concern is the smaller, but still substantial, disagreement between the values of the different expressions at temperatures close to $10^{4} \mathrm{~K}$. In this regime, the differ-

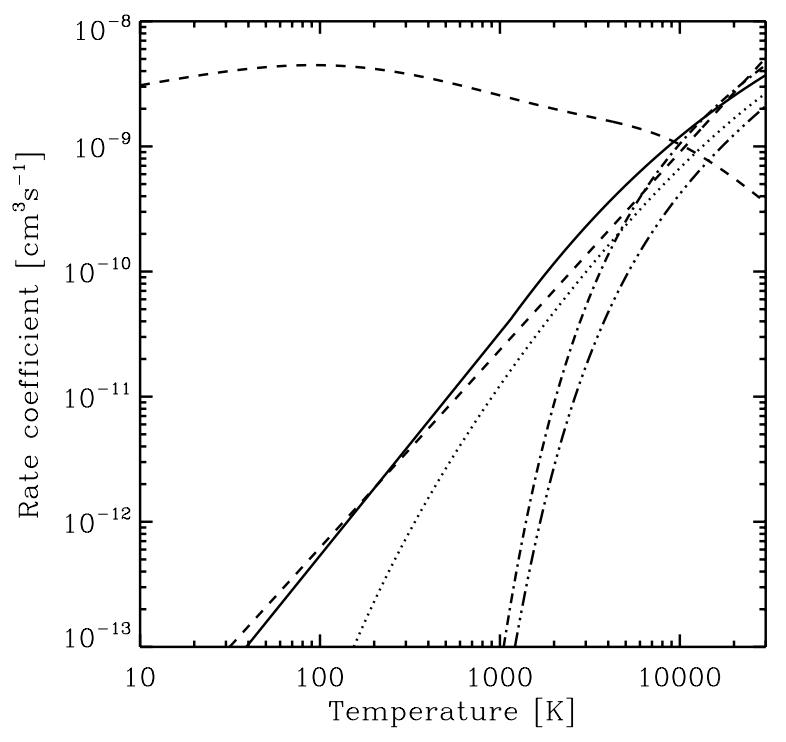

Figure 5. Comparison of different rate coefficients for the collisional detachment of $\mathrm{H}^{-}$by $\mathrm{H}$ (reaction 11). The values shown are taken from Abel et al. (1997) (solid line), our fit to Janev, Reiter \& Samm (2003) (dotted line), Lenzuni, Chernoff \& Salpeter (1991) (lower dashed line) and Browne \& Dalgarno (1969); in the latter case, the dash-dotted line shows the results that they obtain for the Bardsley, Herzenberg \& Mandl (1966a|b) interaction potential, and the dash-dot-dot-dotted line shows their results for the Chen \& Peacher (1968) potential. For comparison, we also plot the rate coefficient for reaction 3 , the associative detachment of $\mathrm{H}^{-}$by $\mathrm{H}$ (upper dashed line). The extremely large uncertainty in the rate of reaction 11 below a few thousand $\mathrm{K}$ is unlikely to significantly affect the chemical evolution of the gas, as in this temperature regime, all of the different expressions yield values that are much smaller than the associative detachment rate.

ent determinations differ by a factor of three to four, and the magnitude of the rate coefficient is similar to that for associative detachment, meaning that this uncertainty potentially has a much larger impact on the $\mathrm{H}^{-}$abundance and the $\mathrm{H}_{2}$ formation rate.

We have explored the effect that the uncertainty in this reaction has on the value of $J_{\text {crit }}$. In runs with a T4 spectrum, we find that the uncertainty introduced into our estimate of $J_{\text {crit }}$ is very small, of the order of a few percent. This is unsurprising, as in these runs, the dominant destruction process for $\mathrm{H}^{-}$is photodissociation and so even large changes in the rate of reaction 11 have little influence on the $\mathrm{H}^{-}$abundance and hence little impact on the $\mathrm{H}_{2}$ formation rate. In runs with a $\mathrm{T} 5$ spectrum, on the other hand, the uncertainty in the rate of reaction 11 has a much larger effect, with $J_{\text {crit }}$ varying between 1630 (if we use the rate from Abel et al. 1997) and 2600 (if we use the rate from Browne \& Dalgarno 1969 obtained with the Chen \& Peacher potential). The total uncertainty in this case is therefore approximately $60 \%$.

Although we would like to be able to recommend a particular choice of rate coefficient for this reaction, it is highly unclear which of the different possibilities is likely to be the most accurate. This process is therefore greatly in need of further theoretical or experimental study in order to 
clarify the behaviour of its rate coefficient at temperatures $T<10^{4} \mathrm{~K}$.

\subsubsection{Collisional ionization of $H$ by $e^{-}$(reaction 12)}

Several different expressions for the rate coefficient of this reaction are in reasonably common usage in the astrophysical literature. Previous studies of the direct collapse model have typically adopted either the fit given in Abel et al. (1997)

$$
\begin{aligned}
k_{12, \mathrm{~A} 97}= & \exp [-32.71396786 \\
& +13.5365560\left(\ln T_{\mathrm{e}}\right) \\
& -5.73932875\left(\ln T_{\mathrm{e}}\right)^{2} \\
& +1.56315498\left(\ln T_{\mathrm{e}}\right)^{3} \\
& -0.28770560\left(\ln T_{\mathrm{e}}\right)^{4} \\
& +3.48255977 \times 10^{-2}\left(\ln T_{\mathrm{e}}\right)^{5} \\
& -2.63197617 \times 10^{-3}\left(\ln T_{\mathrm{e}}\right)^{6} \\
& +1.11954395 \times 10^{-4}\left(\ln T_{\mathrm{e}}\right)^{7} \\
& -2.03914985 \times 10^{-6}\left(\ln T_{\mathrm{e}}\right)^{8} \mathrm{~cm}^{3} \mathrm{~s}^{-1}
\end{aligned}
$$

which is based on data from Janev, Langer \& Evans (1987), or the expression given in Omukai (2001),

$k_{12, \mathrm{O} 01}=4.25 \times 10^{-11} T^{1 / 2} \exp \left(-\frac{157800}{T}\right) \mathrm{cm}^{3} \mathrm{~s}^{-1}$

which comes from Lenzuni, Chernoff \& Salpeter (1991). On the other hand, Hui \& Gnedin (1997) give a different expression

$k_{12, \mathrm{HG} 97}=\frac{21.11}{T^{3 / 2}} e^{-\lambda / 2} \frac{\lambda^{-1.089}}{\left(1+[\lambda / 0.354]^{0.874}\right)^{1.101}} \mathrm{~cm}^{3} \mathrm{~s}^{-1}(23)$

where $\lambda=315614 / T$, which is their fit to Lotz (1967). Finally, Scholz \& Walters (1991) give the expression

$$
\begin{aligned}
k_{12, \mathrm{SW} 91}=\quad & \exp [-96.1443 \\
& +37.9523 \ln T \\
& -7.96885(\ln T)^{2} \\
& +8.83922 \times 10^{-1}(\ln T)^{3} \\
& -5.34513 \times 10^{-2}(\ln T)^{4} \\
& +1.66344 \times 10^{-3}(\ln T)^{5} \\
& -2.08888 \times 10^{-5}(\ln T)^{6} \\
& -157800 / T] \mathrm{cm}^{3} \mathrm{~s}^{-1},
\end{aligned}
$$

which is based on the accurate cross-section measurements of Shah, Elliott \& Gilbody (1987).

The behaviour of these different expressions as a function of temperature is compared in Figure 6. At low temperatures $\left(T \ll 10^{5} \mathrm{~K}\right)$, the collisional ionization rate has an exponential dependence on temperature, $k_{12} \propto e^{-157800 / T}$, since only a small number of electrons in the exponential tail of the Maxwell-Boltzmann distribution have sufficient energy to ionize hydrogen. Therefore, in order to allow us to better see the difference between the different rate coefficients, we do not plot the rate coefficient $k_{12}$ in Figure 6 but instead plot the value of $k_{12} / e^{-157800 / T}$. From the Figure, we see that at temperatures $T>10^{4} \mathrm{~K}$, the different versions of the collisional ionization rate coefficient agree fairly well. In this temperature regime, the different expression have roughly the same temperature dependence but disagree

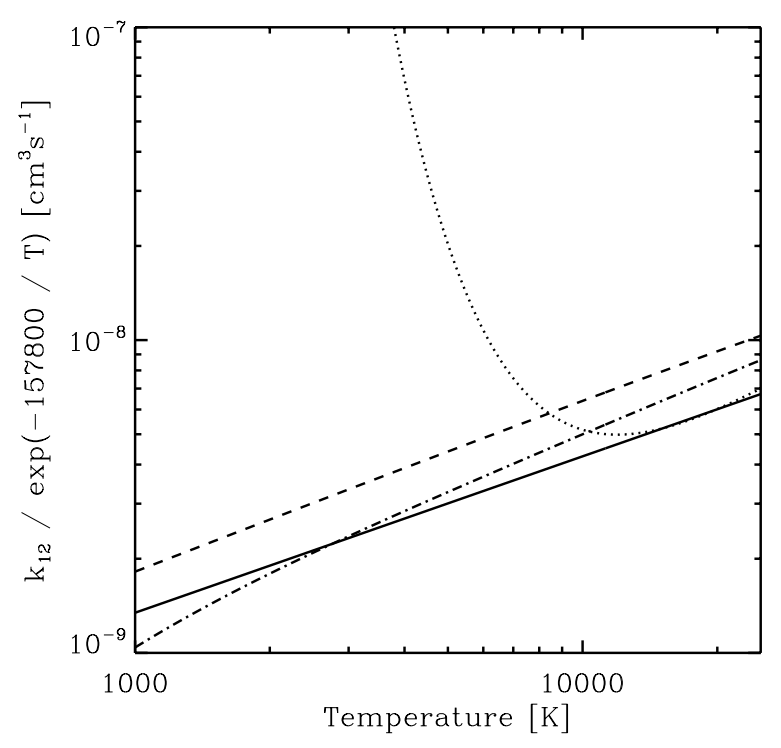

Figure 6. Comparison of different rate coefficients for the collisional ionization of $\mathrm{H}$ by electrons (reaction 12). To highlight the difference between the various rate coefficients, we remove the underlying exponential temperature dependence by plotting the quantity $k_{12} / e^{-157800 / T}$, where $k_{12}$ is the rate coefficient. The values shown in the plot come from Lenzuni, Chernoff \& Salpeter (1991) (solid line) Abel et al. (1997) (dotted line), Hui \& Gnedin 1997) (dashed line) and Scholz \& Walters (1991) (dash-dotted line).

on the normalization of the rate coefficient by around $30 \%$. At lower temperatures, the rates from Lenzuni, Chernoff \& Salpeter (1991), Scholz \& Walters (1991) and Hui \& Gnedin (1997) continue to agree fairly well, while the Abel et al. (1997) rate begins to disagree strongly with the others. It seems likely that this is due to a problem with the Abel et al. fit at these low temperatures, although it should be remembered that this occurs in a regime where the value of $k_{12}$ is falling off exponentially with decreasing temperature, and so for most applications, the Abel et al. rate remains a suitable choice.

We have determined the value of $J_{\text {crit }}$ that we obtain when using each of these four expressions for the rate coefficient. In runs with a T4 spectrum, we find that the values all lie in the range $J_{\text {crit }}=16.2-18.0$, while in the case of a T5 spectrum, the corresponding range of values is $J_{\text {crit }}=1460$ 1630. The uncertainty in the rate of reaction 12 therefore introduces no more than a $10 \%$ uncertainty into our estimates of $J_{\text {crit }}$.

Finally, which of these rate coefficients should one actually use in future calculations? Since the total uncertainty (statistical and systematic) in the Shah, Elliott \& Gilbody (1987) measurements is unlikely to exceed $20 \%$ (see e.g. the discussion in Barklem et al. 2011) and they are also in good agreement with available high-accuracy theoretical calculations (e.g. Bray \& Stelbovics 1993), we recommend the use of the rate coefficient based on their measurements, i.e. the expression given in Scholz \& Walters (1991). 


\subsubsection{Mutual neutralization of $\mathrm{H}^{-}$with $\mathrm{H}^{+}$(reaction 22)}

In our fiducial model, we use the rate coefficient derived by Croft, Dickinson \& Gadea (1999), based on the crosssections of Fussen \& Kubach (1986). This is well fit by the simple function

$k_{22, \mathrm{C} 99}=2.4 \times 10^{-6} T^{-1 / 2}\left(1+\frac{T}{20000}\right) \mathrm{cm}^{3} \mathrm{~s}^{-1}$.

More recently, Urbain et al. (2012) state that their merged beam measurements "support the rate coeffcient compiled by Croft et al.", but do not give numerical details of their measured cross-sections, meaning that it is not possible to assess how accurately their measurements agree with the earlier Fussen \& Kubach (1986) cross-sections. The rate coefficient for reaction 22 has also been calculated relatively recently by Stenrup, Larson \& Elander (2009). Their results are well fit in the temperature range $10<T<10000 \mathrm{~K}$ by the function

$$
\begin{aligned}
k_{22, \text { SLE09 }}= & 2.96 \times 10^{-6} T^{-1 / 2}-1.73 \times 10^{-9} \\
& +2.50 \times 10^{-10} T^{1 / 2} \\
& -7.77 \times 10^{-13} \mathrm{~T} \mathrm{~cm}^{3} \mathrm{~s}^{-1}
\end{aligned}
$$

We compare this with the Croft et al. rate in Figure 7, where we plot the ratio $k_{22, \text { SLE09 }} / k_{22 \text {, C99 }}$ for temperatures in the range $10<T<10000 \mathrm{~K}$. We see that the Stenrup et al. rate coefficient is higher than the Croft et al. rate coefficient at all temperatures, by around $20-25 \%$. We have investigated the effect of using the Stenrup et al. rate coefficient in place of the Croft et al. rate coefficient in our models. We find that if we do so, then there is essentially no change in the value of $J_{\text {crit }}$ we recover for runs performed using a T4 spectrum. In runs with a T5 spectrum, however, we find that $J_{\text {crit }}=1630$ when we use the Croft et al. rate coefficient and $J_{\text {crit }}=1550$ when we use the Stenrup et al. rate coefficient. The current uncertainty in the value of the mutual neutralization rate coefficient therefore introduces an uncertainty of around $5 \%$ into our derived value of $J_{\text {crit }}$.

We note however that a number of treatments of primordial chemistry in the literature use expressions for this rate coefficient based either on the measurements of Moseley et al. (1970) or taken from the review of Dalgarno \& Lepp (1987). These differ from the Croft et al. rate coefficient by factors of a few, as summarized in Glover, Savin \& Jappsen (2006). We have explored the effect on $J_{\text {crit }}$ of using one of these older values for the rate coefficient, and find that if we use the Moseley et al. value, we obtain $J_{\text {crit }}=17.4$ for a T4 spectrum and $J_{\text {crit }}=1070$ for a T5 spectrum, while if we use the Dalgarno \& Lepp value, we obtain instead $J_{\text {crit }}=18.3$ for a T4 spectrum and $J_{\text {crit }}=1940$ for a T5 spectrum. We therefore see that the use of an older value for this rate coefficient can introduce an error of up to $\sim 30 \%$ into the value we derive for $J_{\text {crit }}$.

Because of this, and in view of the fact that the most recent determinations of the rate coefficient for reaction 22 agree well with the Croft et al. value and disagree significantly with the Moseley et al. (1970) and Dalgarno \& Lepp (1987) values, we recommend that the rate coefficients for this reaction from the latter two sources should no longer be used when studying the direct collapse model.

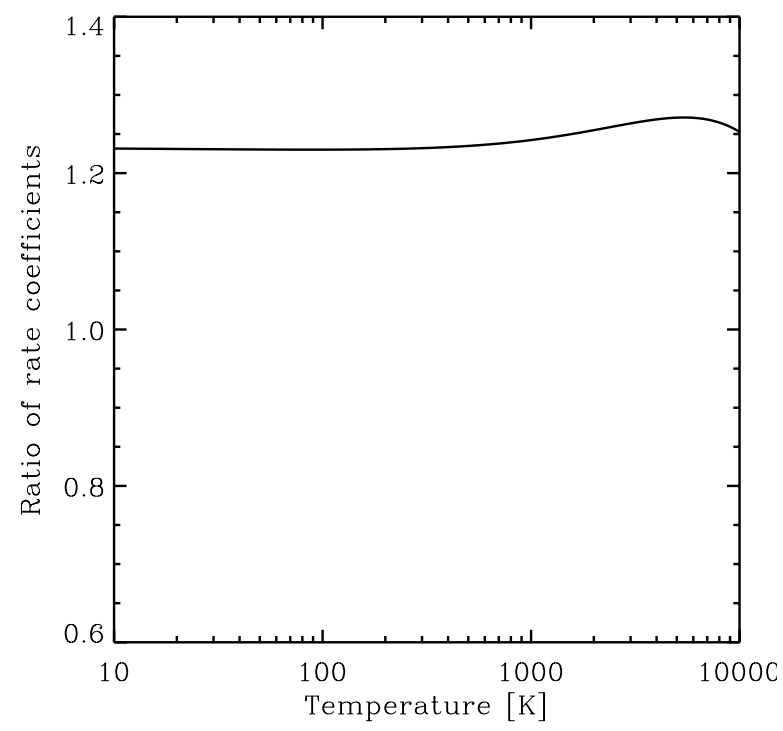

Figure 7. Ratio of the rate coefficient for the mutual neutralization of $\mathrm{H}^{-}$by $\mathrm{H}^{+}$(reaction 22) given by Stenrup, Larson \& Elander (2009) to that given by Croft, Dickinson \& Gadea (1999).

\section{IMPACT OF COOLING RATE UNCERTAINTIES ON $J_{\mathrm{CRIT}}$}

The cooling function that we use in our one-zone calculations accounts for the effects of radiative cooling from a large number of different coolants, including atomic hydrogen, atomic helium, $\mathrm{H}_{2}, \mathrm{H}_{2}^{+}$, and $\mathrm{HeH}^{+}$(see the detailed discussion in Glover \& Savin 2009). However, in practice, the only coolants that contribute substantially to the overall cooling rate are atomic hydrogen and $\mathrm{H}_{2}$. We therefore focus our attention on these two coolants and do not consider the effects of uncertainties in the other cooling rates, as these introduce a negligible uncertainty into the total cooling rate.

\section{1 $\quad \mathrm{H}_{2}$ cooling}

There are two main sources of uncertainty that we need to consider in the case of $\mathrm{H}_{2}$ cooling. At low densities, the cooling rate scales linearly with the collisional excitation rate, which is given by the sum of a series of contributions corresponding to collisions between $\mathrm{H}_{2}$ and $\mathrm{H}, \mathrm{H}_{2}$ and $\mathrm{He}, \mathrm{H}_{2}$ and $\mathrm{H}^{+}$, etc. In this regime, uncertainties in these contributions directly affect the $\mathrm{H}_{2}$ cooling rate. At high densities, on the other hand, the $\mathrm{H}_{2}$ level populations reach LTE. In this regime, the $\mathrm{H}_{2}$ cooling rate is insensitive to the collisional excitation rate, and depends only on the partition function for the $\mathrm{H}_{2}$ molecule, the energies of the various excited rotational and vibrational levels, and their spontaneous radiative de-excitation rates. All of these quantities are known accurately, and so in principle there should be almost no uncertainty in the $\mathrm{H}_{2}$ cooling rate in these conditions. In practice, however, the $\mathrm{H}_{2}$ cooling rate in the LTE limit is often represented by a simple analytical function of temperature that approximates the true $\mathrm{H}_{2}$ cooling rate. Any error in this approximation therefore translates into an un- 
Table 2. Sensitivity of $J_{\text {crit }}$ to the rate coefficients adopted for the listed cooling processes

\begin{tabular}{lcc}
\hline Process & Sensitivity (T4) & Sensitivity (T5) \\
\hline Lyman- $\alpha$ & 1.04 & 1.54 \\
$\mathrm{H}_{2}:$ collisions with $\mathrm{H}$ & 10.3 & 4.53 \\
$\mathrm{H}_{2}$ : collisions with $\mathrm{He}$ & 1.05 & 1.03 \\
$\mathrm{H}_{2}$ : collisions with $\mathrm{H}_{2}$ & 1.00 & 1.00 \\
$\mathrm{H}_{2}$ : collisions with $\mathrm{H}^{+}$ & 1.00 & 1.00 \\
$\mathrm{H}_{2}$ : collisions with $\mathrm{e}^{-}$ & 1.00 & 1.00 \\
\hline
\end{tabular}

certainty in the resulting parameterization of the $\mathrm{H}_{2}$ cooling rate.

\subsubsection{Low density limit}

In primordial gas, the most important collision partners responsible for exciting the internal energy levels of the $\mathrm{H}_{2}$ molecule are $\mathrm{H}$ and $\mathrm{He}$ atoms, other $\mathrm{H}_{2}$ molecules, protons and electrons (Glover \& Abel 2008). In order to quantify which of these possible collisions partners is most important in the present case, we have carried out a similar analysis to that described in Section 3.1 We constructed a series of different variants of our cooling model by adjusting the individual collisional rate coefficients either upwards or downwards by a factor of $10^{0.5}$ from their fiducial values ${ }^{1}$ We next used our variant models to determine $J_{\text {crit }}$ and then examined the difference in $J_{\text {crit }}$ that resulted from adjusting each rate coefficient either upwards or downwards. Finally, by taking the ratio of the largest and the smallest values of $J_{\text {crit }}$ that we obtained when modifying the rate of a particular collision process, we could define a sensitivity for that process, analogous to the sensitivities determined for the different chemical rate coefficients in Section 3 .

The results of our analysis are summarized in Table 2 We see that $J_{\text {crit }}$ displays a high sensitivity to the rate of only one of our considered collisional excitation processes, the excitation of $\mathrm{H}_{2}$ by collisions with $\mathrm{H}$ atoms. This strongly suggests that at the densities and temperatures where $J_{\text {crit }}$ is determined, collisions between $\mathrm{H}_{2}$ and $\mathrm{H}$ dominate the total $\mathrm{H}_{2}$ cooling rate.

This behaviour differs from that found in metal-free protogalaxies that are not illuminated by extremely strong radiation fields, as in these systems $\mathrm{H}_{2}-\mathrm{He}$ collisions play an important role, as do $\mathrm{H}_{2}-\mathrm{H}^{+}$and $\mathrm{H}_{2}-\mathrm{e}^{-}$collisions if the gas is cooling from an initially ionized state (Glover \& Abel 2008). However, this difference is easy to understand. As we have already seen in paper I, in atomic cooling haloes illuminated by a radiation field with $J_{21} \sim J_{\text {crit }}$, the critical factor that determines whether or not the gas can cool is the behaviour of the $\mathrm{H}_{2}$ fraction at densities $n \sim 10^{3} \mathrm{~cm}^{-3}$ and temperatures $T \sim 8000 \mathrm{~K}$. In these conditions, the gas is mostly atomic and the fractional ionization is low, $x_{\mathrm{H}^{+}} \sim 10^{-4}$. If we compare the mean $\mathrm{H}_{2}$ cooling rates per collision due to collisions with $\mathrm{H}, \mathrm{He}, \mathrm{H}^{+}$and $\mathrm{e}^{-}$at this temperature and density, then we find that $\Lambda_{\mathrm{H}_{2}, \mathrm{H}} / \Lambda_{\mathrm{H}_{2}, \mathrm{He}} \sim 4$

1 The latter were taken from Glover \& Abel (2008), although the rates for $\mathrm{H}_{2}-\mathrm{H}^{+}$and $\mathrm{H}_{2}-\mathrm{e}^{-}$collisions were updated to account for new data, as summarized in Appendix A of Paper I. and $\Lambda_{\mathrm{H}_{2}, \mathrm{H}} / \Lambda_{\mathrm{H}_{2}, \mathrm{H}^{+}} \sim \Lambda_{\mathrm{H}_{2}, \mathrm{H}} / \Lambda_{\mathrm{H}_{2}, \mathrm{e}^{-}} \sim 0.3$. Since the He:H ratio (by number) in primordial gas is approximately 0.08 , it is clear that collisions with helium contribute only a few percent of the total $\mathrm{H}_{2}$ cooling rate, and that the contributions from collisions with protons and electrons are negligible. In the scenario considered by Glover \& Abel (2008), gas cools and recombines from an initially ionized state, but there is no ultraviolet background. In this case, $\mathrm{H}_{2}$ cooling becomes effective much earlier in the collapse, when the fractional ionization is significantly larger. In addition, Glover \& Abel (2008) were primarily concerned with the behaviour of the cooling rate at low temperatures, where the difference between the contribution from $\mathrm{H}_{2}-\mathrm{H}$ collisions and from collisions with $\mathrm{He}, \mathrm{H}^{+}$or electrons is much more pronounced.

Since our analysis demonstrates that it is essentially just the $\mathrm{H}_{2}-\mathrm{H}$ collisional excitation rate that plays a role in determining $J_{\text {crit }}$, we choose to focus our attention on this process, and will not discuss any uncertainties that may exist in the other collisional excitation rates. In our default model, the treatment we use for the $\mathrm{H}_{2}-\mathrm{H}$ contribution to the $\mathrm{H}_{2}$ cooling rate is taken from Glover \& Abel (2008). They give the following analytic fit to the $\mathrm{H}_{2}$ cooling rate, valid in the temperature range $1000<T<6000 \mathrm{~K}$ :

$$
\begin{aligned}
\Lambda_{\mathrm{H}_{2}, \mathrm{GA} 08}= & \operatorname{dex}[-24.311209 \\
& +4.6450521\left(\log T_{3}\right) \\
& -3.7209846\left(\log T_{3}\right)^{2} \\
& +5.9369081\left(\log T_{3}\right)^{3} \\
& -5.5108047\left(\log T_{3}\right)^{4} \\
& \left.+1.5538288\left(\log T_{3}\right)^{5}\right] \mathrm{erg} \mathrm{s}^{-1} \mathrm{~cm}^{3},
\end{aligned}
$$

where $T_{3}=T / 1000 \mathrm{~K}{ }^{2}$ This fit is based on the set of collisional excitation rate coefficients computed by Wrathmall \& Flower (2007; see also Wrathmall, Gusdorf \& Flower 2007). These authors use a fully quantal treatment of the $\mathrm{H}_{2}-\mathrm{H}$ system in their calculations and make use of the potential energy surface of Mielke, Garrett \& Peterson (2002).

The other main treatment of $\mathrm{H}_{2}$ cooling that is in widespread use in numerical models of primordial gas is that of Galli \& Palla (1998). They give the following analytical fit to the $\mathrm{H}_{2}-\mathrm{H}$ cooling rate:

$$
\begin{aligned}
\Lambda_{\mathrm{H}_{2}, \mathrm{GP} 98}= & \operatorname{dex}\left[-103.0+97.59 \log T-48.05(\log T)^{2}\right. \\
& -48.05(\log T)^{2}+10.80(\log T)^{3} \\
& \left.-0.9032(\log T)^{4}\right] \mathrm{erg} \mathrm{s}^{-1} \mathrm{~cm}^{3}
\end{aligned}
$$

This fit is based on the quantal calculations of Forrey et al. (1997) at $T<600 \mathrm{~K}$ and the quasi-classical calculations of Mandy \& Martin (1993) at $T>600 \mathrm{~K}$. In both cases, older versions of the $\mathrm{H}_{2}-\mathrm{H}$ potential energy surface were used, as summarized in Galli \& Palla (1998).

Finally, an alternative version of the $\mathrm{H}_{2}-\mathrm{H}$ cooling rate is given in Martin, Schwarz \& Mandy (1996). These authors present a complicated fit to the $\mathrm{H}_{2}$ cooling rate in the regime where $\mathrm{H}$ collisions dominate that is valid over a wide range of different temperatures. However, the low density limit of

\footnotetext{
2 Glover \& Abel (2008) also give fits for $\Lambda_{\mathrm{H}_{2}}$ in the temperature ranges $10<T<100 \mathrm{~K}$ and $100<T<1000 \mathrm{~K}$, but the behaviour of the $\mathrm{H}_{2}$ cooling function at these temperatures has no influence on the value of $J_{\text {crit }}$.
} 
this fit is easy to extract and can be written as

$$
\begin{aligned}
\Lambda_{\mathrm{H}_{2}, \text { MSM96 }}= & \operatorname{dex}[+195.09-137.9986 \log T \\
& +29.802(\log T)^{2} \\
& -2.165163(\log T)^{3} \\
& +91.60106 \log (1.0+2680.075 / T) \\
& -105.7438 \log (1.0+2889.762 / T) \\
& -4328.147 / T] \mathrm{erg} \mathrm{s}^{-1} \mathrm{~cm}^{3} .
\end{aligned}
$$

This version of the cooling rate is also based on the calculations of Mandy \& Martin (1993).

In the upper panel of Figure 8, we compare the temperature dependence of these three versions of the $\mathrm{H}_{2}-\mathrm{H}$ cooling rate. We see that although the different rates disagree somewhat at temperatures close to $1000 \mathrm{~K}$, there is good agreement in the temperature range $T \sim 6000-8000 \mathrm{~K}$ relevant for $J_{\text {crit. }}$. At even higher temperatures, $T>10^{4} \mathrm{~K}$, there is significant disagreement between the rates, but this is simply a consequence of the breakdown of the Glover \& Abel (2008) and Galli \& Palla (1998) analytical fits in this temperature regime; as previously noted, the Glover \& Abel fit is formally only valid at $T<6000 \mathrm{~K}$, while the Galli \& Palla fit is valid only for $T<10^{4} \mathrm{~K}$. Fortunately, at these very high temperatures, the precise behaviour of $\Lambda_{\mathrm{H}_{2}, \mathrm{H}}$ is unlikely to be important, as Lyman- $\alpha$ cooling dominates in this temperature regime.

As the $\mathrm{H}_{2}$ cooling rate varies over several orders of magnitude in the temperature range that we are examining, it is difficult to tell from the upper panel of Figure 8 exactly how well the different cooling rates agree at $T \sim 6000-8000 \mathrm{~K}$. Therefore, in the lower panel of the Figure, we plot instead the ratio of the Galli \& Palla rate to the Glover \& Abel rate (solid line) and the ratio of the Martin, Schwarz \& Mandy rate to the Glover \& Abel rate (dashed line). We see that at the temperatures of interest, all three rates agree to within around $10 \%$.

We have investigated the impact of this small uncertainty on $J_{\text {crit }}$. We find that in runs with a T4 spectrum, it introduces an uncertainty of roughly $10 \%$ into our determination of $J_{\text {crit }}$, while in runs with a T5 spectrum, the corresponding uncertainty is $\sim 5 \%$.

\subsubsection{LTE limit}

In the LTE limit, the $\mathrm{H}_{2}$ cooling rate can be computed very accurately, since it only depends on the energies and radiative de-excitation rates of the rotational and vibrational levels of $\mathrm{H}_{2}$, all of which are known with a high degree of precision. Glover (2011) numerically evaluated the LTE cooling rate and produced the following analytical fit for the cooling rate coefficient, which is valid in the temperature range $100<T<10000 \mathrm{~K}$ and has an error of no more than $5 \%$ over this range:

$$
\begin{aligned}
\Lambda_{\mathrm{H}_{2}, \mathrm{LTE}, \mathrm{G} 11}= & \operatorname{dex}[-20.584225 \\
& +5.0194035 \log T_{3} \\
& -1.5738805\left(\log T_{3}\right)^{2} \\
& -4.7155769\left(\log T_{3}\right)^{3} \\
& +2.4714161\left(\log T_{3}\right)^{4} \\
& +5.4710750\left(\log T_{3}\right)^{5}
\end{aligned}
$$
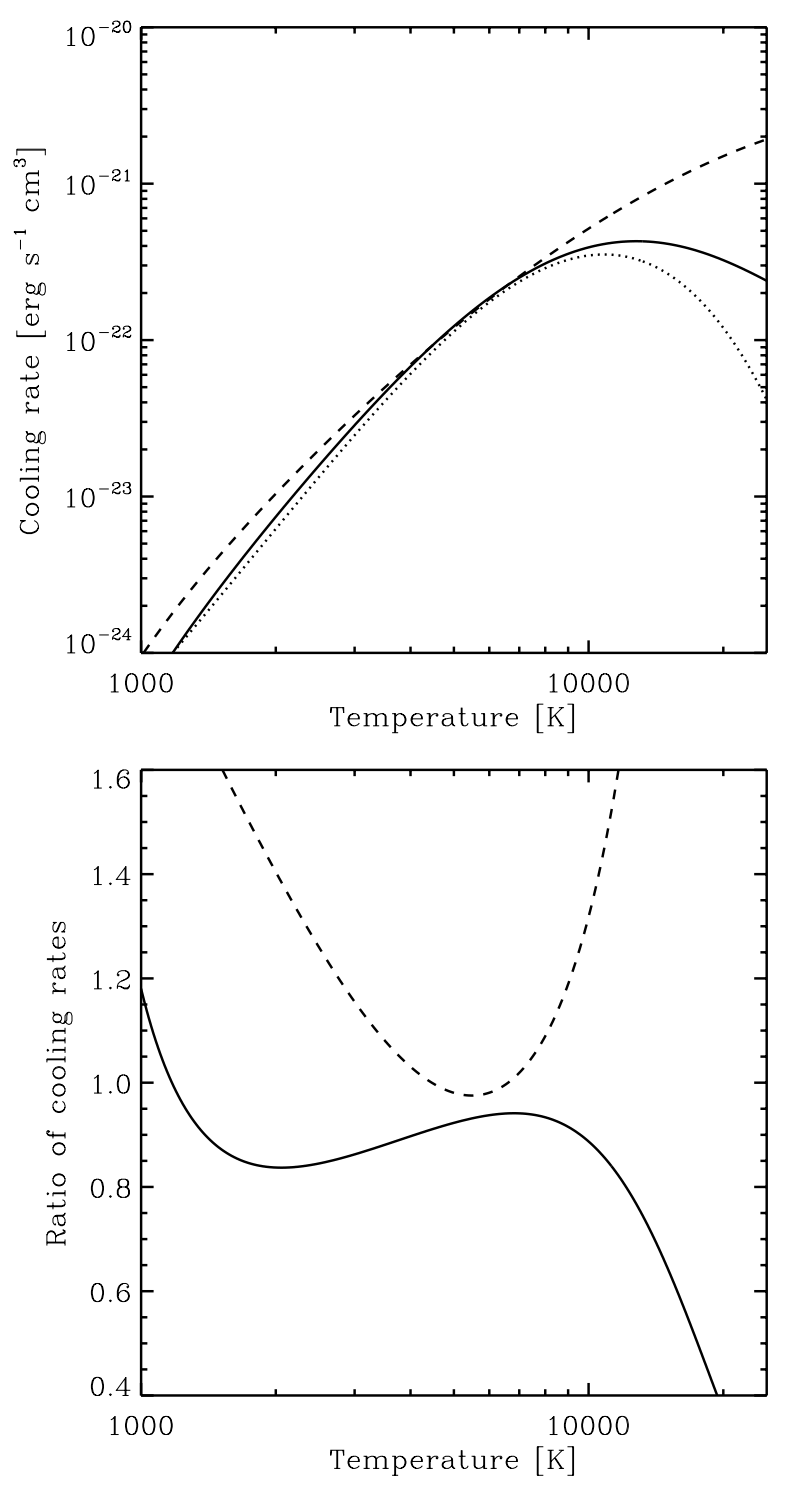

Figure 8. Upper panel: comparison of $\mathrm{H}_{2}-\mathrm{H}$ cooling rate coefficients. The solid line shows the high temperature fit from Glover \& Abel (2008), the dotted line shows the expression from Galli \& Palla (1998) and the dashed line shows the expression from Martin, Schwarz \& Mandy (1996). The significant disagreement in behaviour at $T>10^{4} \mathrm{~K}$ is a consequence of the fact that the fits given in Glover \& Abel (2008) and Galli \& Palla (1998) are not valid at these temperatures. Lower panel: ratio of the Galli \& Palla (1998) rate to the Glover \& Abel (2008) rate (solid line) and of the Martin, Schwarz \& Mandy (1996) rate to the Glover \& Abel (2008) rate (dashed line).

$$
\begin{aligned}
& -3.9467356\left(\log T_{3}\right)^{6} \\
& -2.2148338\left(\log T_{3}\right)^{7} \\
& \left.+1.8161874\left(\log T_{3}\right)^{8}\right] \quad \operatorname{erg~s}^{-1} .
\end{aligned}
$$

However, many treatments of $\mathrm{H}_{2}$ cooling in primordial gas (see e.g. Galli \& Palla 1998, Bryan et al. 2014, Grassi et al. 2014) continue to make use of the approximate analytical expression for the $\mathrm{H}_{2}$ LTE cooling rate given in Hollenbach \& McKee (1979):

$\Lambda_{\mathrm{H}_{2}, \mathrm{LTE}, \mathrm{HM} 79}=\Lambda_{\mathrm{H}_{2}, \mathrm{rot}}+\Lambda_{\mathrm{H}_{2}, \mathrm{vib}}$ 


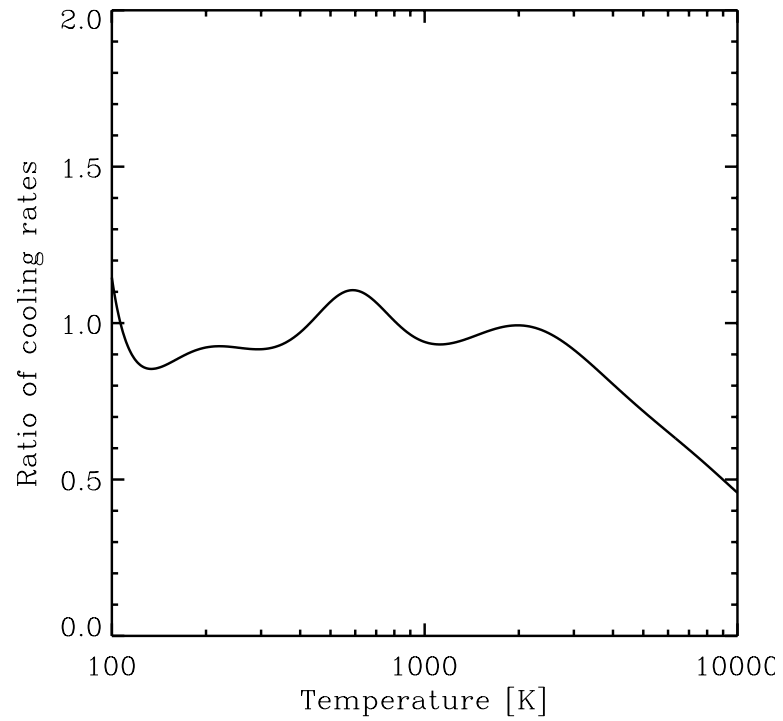

Figure 9. Ratio of the accurate $\mathrm{H}_{2}$ LTE cooling rate from Glover (2011) to the approximate analytical fit given in Hollenbach \& McKee (1979).

where

$$
\begin{aligned}
\Lambda_{\mathrm{H}_{2}, \text { rot }}= & \left(\frac{9.5 \times 10^{-22} T_{3}^{3.76}}{1+0.12 T_{3}^{2.1}}\right) \exp \left[-\left(0.13 / T_{3}\right)^{3}\right] \\
& +3 \times 10^{-24} \exp \left(-0.51 / T_{3}\right) \mathrm{erg} \mathrm{s}^{-1},
\end{aligned}
$$

and

$$
\begin{aligned}
\Lambda_{\mathrm{H}_{2}, \mathrm{vib}}= & 6.7 \times 10^{-19} \exp \left(-5.86 / T_{3}\right) \\
+ & 1.6 \times 10^{-18} \exp \left(-11.7 / T_{3}\right) \mathrm{erg} \mathrm{s}^{-1} .
\end{aligned}
$$

We compare these two cooling rates in Figure 9, where we plot their ratio, $\Lambda_{\mathrm{H}_{2}, \mathrm{LTE}, \mathrm{G} 11} / \Lambda_{\mathrm{H}_{2}, \mathrm{LTE}, \mathrm{HM} 79}$. We see that at temperatures below $T \sim 2000 \mathrm{~K}$, the two rates agree reasonably well, with differences of no more than $10 \%$. At higher temperatures, however, the expression from Hollenbach \& McKee (1979) yields a larger cooling rate than the one from Glover (2011), with the discrepancy worsening to as much as a factor of two as we near $10^{4} \mathrm{~K}$.

In practice, this discrepancy does not have a large impact on our estimate of $J_{\text {crit }}$, as this is determined at densities somewhat lower than the $\mathrm{H}_{2}$ critical density. If we compare the results of runs performed using the Glover (2011) LTE rate with those performed using the Hollenbach \& McKee (1979) rate, we find that $J_{\text {crit }}$ differs by around $10 \%$. This holds regardless of whether we use a T4 or a T5 spectrum.

\subsection{Lyman- $\alpha$ cooling}

\subsubsection{Collisions with electrons}

Most numerical models of primordial gas use the following expression for the cooling rate coefficient arising from $\mathrm{H}-\mathrm{e}^{-}$ collisions (commonly referred to simply as Lyman- $\alpha$ cooling):

$\Lambda_{\mathrm{H}, \mathrm{C} 92}=\left(\frac{7.5 \times 10^{-19}}{1+T_{5}^{1 / 2}}\right) e^{-118348 / T} \mathrm{erg} \mathrm{s}^{-1} \mathrm{~cm}^{3}$

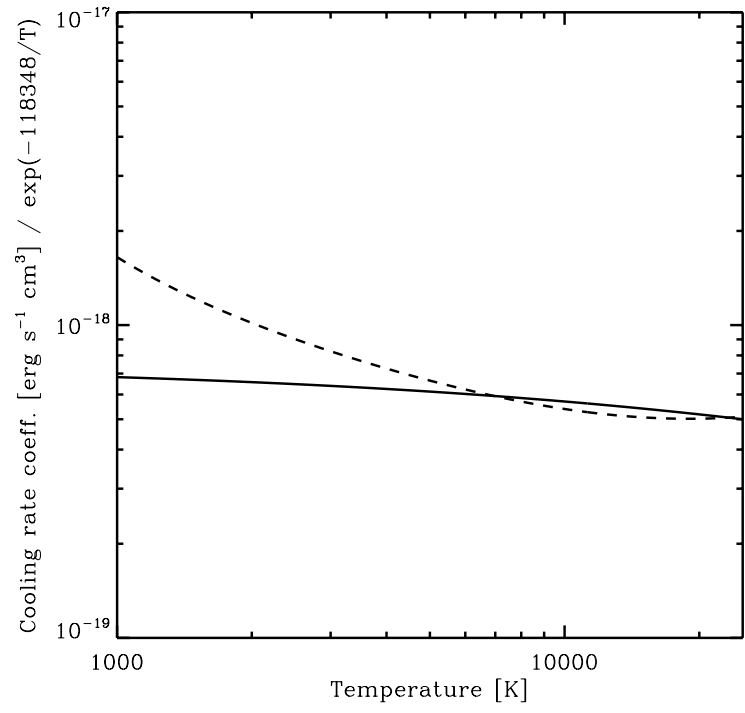

Figure 10. Comparison of the Lyman- $\alpha$ cooling rate coefficients from Cen (1992) (solid line) and Scholz \& Walters (1991) (dashed line). Both rate coefficients have been divided by a factor $e^{-118348 / T}$ in order to remove the underlying exponential dependence on temperature, allowing us to more clearly see the difference in behaviour between the two expressions.

where $T_{5}=T / 10^{5} \mathrm{~K}$. This expression comes from Cen (1992), and is a minor modification of the cooling rate coefficient originally given in Black (1981). In order to assess the level of uncertainty in this parameterization of the cooling rate, we compare it with the expression given in Scholz \& Walters (1991):

$\Lambda_{\mathrm{H}, \mathrm{SW} 91}=10^{-20} f(T) e^{-118348 / T} \mathrm{erg} \mathrm{s}^{-1} \mathrm{~cm}^{3}$,

where

$$
\begin{aligned}
f(T)= & \exp [213.7913-113.9492 \ln T \\
& +25.06062(\ln T)^{2} \\
& -2.762755(\ln T)^{3} \\
& +0.1515352(\ln T)^{4} \\
& \left.-3.290382 \times 10^{-3}(\ln T)^{5}\right] .
\end{aligned}
$$

These two versions of the Lyman- $\alpha$ cooling rate are compared in Figure 10. To enable us to more clearly distinguish the differences in the behaviour of the two rates, we have divided both of them by a factor $e^{-118348 / T}$ in order to take out the underlying exponential dependence on temperature. We see that although there is some disagreement in the rates at low temperatures (where Lyman- $\alpha$ cooling is unimportant), at the temperatures relevant for $J_{\text {crit }}$ these two different prescriptions agree to within a few percent. This small difference introduces an uncertainty of around $1 \%$ into our estimate of $J_{\text {crit }}$.

\subsubsection{Collisions with $H$ and He atoms}

Electronic states of atomic hydrogen with $n>2$ can also be excited by collisions with $\mathrm{H}$ and $\mathrm{He}$ atoms. The rate coefficients for these processes are much smaller than for $\mathrm{H}-\mathrm{e}^{-}$ collisions, but as in the case of the collisional ionization of 
hydrogen, it is conceivable that these processes may become important if the fractional ionization of the gas is very small.

At the temperatures of interest in this study, the atomic hydrogen cooling rate is dominated by the excitation of the $2 p$ and $2 s$ levels, and so there is no need for us to consider excitation to states with $n \geqslant 3$. We can therefore write the cooling rate coefficient that represents the effect of $\mathrm{H}-\mathrm{H}$ collisions as

$\Lambda_{\mathrm{H}, \mathrm{H}}=10.2 \mathrm{eV} \times q_{12}, \mathrm{erg} \mathrm{s}^{-1} \mathrm{~cm}^{3}$,

where $q_{12}$ is the collisional excitation rate coefficient for transitions from $n=1$ to $n=23^{3}$

As in the case of $\mathrm{H}-\mathrm{H}$ collisional ionization, there are very few studies of $\mathrm{H}-\mathrm{H}$ collisional excitation that consider the low energies relevant for our present study. One possibility is the rate given in Lenzuni, Chernoff \& Salpeter (1991), which is based on the work of Drawin (1969):

$$
\begin{aligned}
q_{12}= & 5.8 \times 10^{-15} T^{1 / 2}\left(1.0+1.69 \times 10^{-5} T\right) \\
& \times \exp \left(-\frac{118348}{T}\right) \mathrm{cm}^{3} \mathrm{~s}^{-1},
\end{aligned}
$$

and so

$$
\begin{aligned}
\Lambda_{\mathrm{H}, \mathrm{H}, \mathrm{LCS} 91}= & 9.5 \times 10^{-26} T^{1 / 2}\left(1.0+1.69 \times 10^{-5} T\right) \\
& \times \exp \left(-\frac{118348}{T}\right) \mathrm{erg} \mathrm{s}^{-1} \mathrm{~cm}^{3} .
\end{aligned}
$$

Collisional excitation of $\mathrm{H}$ by $\mathrm{H}$ is also treated by Soon (1992). However, in this case we encounter the same problem as we did when examining the treatment of $\mathrm{H}-\mathrm{H}$ collisional ionization by the same author: the energy threshold adopted in Soon's calculations is only half of the value that it should be (Barklem 2007). If we correct for this problem and numerically integrate the cross-section given in Soon (1992) using the correct energy threshold, then the cooling rate coefficient that we obtain can be fit to within $10 \%$ over the temperature range $1000<T<20000 \mathrm{~K}$ by the function

$\Lambda_{\mathrm{H}, \mathrm{H}, \mathrm{S} 92 \mathrm{~b}}=5.8 \times 10^{-22} T^{-0.75} \exp \left(-\frac{118348}{T}\right) \mathrm{erg} \mathrm{s}^{-1} \mathrm{~cm}^{3}(40)$

In Figure 11. we compare these two different H-H cooling rate coefficients. At the temperatures relevant for $J_{\text {crit }}$, the rate coefficient from Lenzuni, Chernoff \& Salpeter (1991) predicts about an order of magnitude more cooling than the rate coefficient based on Soon (1992). If we compare these values to the $\mathrm{H}_{-} \mathrm{e}^{-}$cooling rate coefficients discussed earlier, then we find that $\Lambda_{\mathrm{H}, \mathrm{H}} / \Lambda_{\mathrm{H}, \mathrm{e}^{-}} \sim 10^{-5}$ for the Lenzuni, Chernoff \& Salpeter (1991) rate coefficient and $\sim 10^{-6}$ for the rate coefficient based on Soon (1992). As the actual fractional ionization of the gas in the conditions where $J_{\text {crit }}$ is determined is around $x \sim 4 \times 10^{-5}$ (see e.g. Paper I, Figure 1), it is unsurprising that if we include the effect of $\mathrm{H}-\mathrm{H}$ cooling using either of the two rate coefficients from the literature, we find that the effect on $J_{\text {crit }}$ is negligible. The large uncertainty in the $\mathrm{H}-\mathrm{H}$ cooling rate therefore has no influence on the value of $J_{\text {crit }}$.

As far as the excitation of $\mathrm{H}$ by collisions with He atoms is concerned, Lenzuni, Chernoff \& Salpeter (1991) give the

\footnotetext{
3 In principle, one should distinguish between the $1 s \rightarrow 2 s$ and $1 s \rightarrow 2 p$ transitions, but in practice, treatments of this process in the literature often assume that both transitions share the same rate coefficient.
}

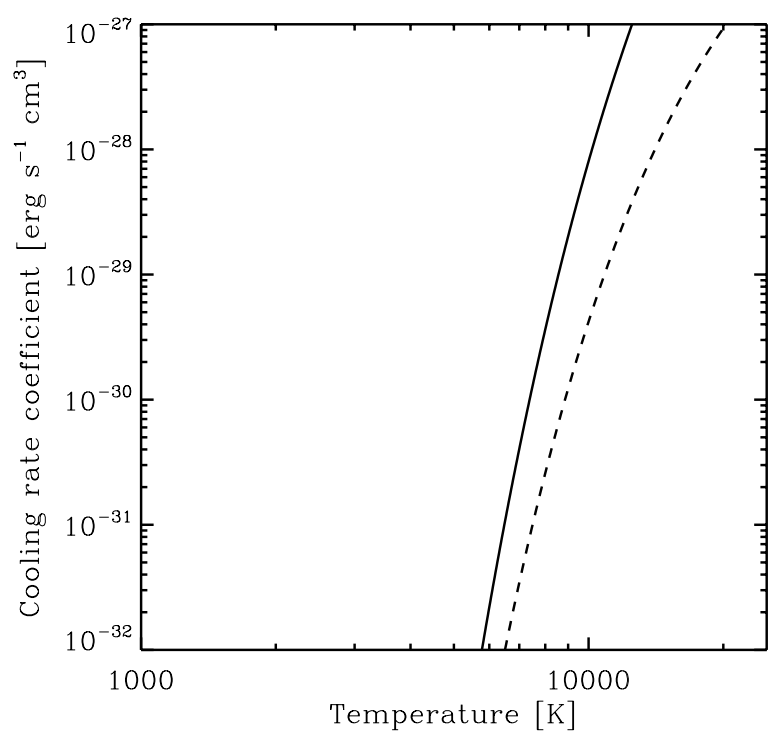

Figure 11. Comparison of the $\mathrm{H}-\mathrm{H}$ collisional excitation cooling rate coefficients from Lenzuni, Chernoff \& Salpeter 1991) (solid line) and Soon (1992) (dashed line).

following expressions for the collisional excitation rates of the $1 s \rightarrow 2 p$ and $1 s \rightarrow 2 s$ transitions respectively:

$$
\begin{aligned}
& q_{1 \rightarrow 2 \mathrm{p}}=1.2 \times 10^{-14} T^{1.2} \exp \left(-\frac{196250}{T}\right) \mathrm{cm}^{3} \mathrm{~s}^{-1}, \\
& q_{1 \rightarrow 2 \mathrm{~s}}=1.2 \times 10^{-15} T^{1.2} \exp \left(-\frac{196250}{T}\right) \mathrm{cm}^{3} \mathrm{~s}^{-1} .
\end{aligned}
$$

These collisional excitation rates are based on the authors' fits to the data of Grosser \& Krüger (1984). Using these excitation rates, we can easily derive the corresponding cooling rate coefficient:

$\Lambda_{\mathrm{H}, \mathrm{He}}=2.2 \times 10^{-25} T^{1.2} \exp \left(-\frac{196250}{T}\right) \mathrm{erg} \mathrm{s}^{-1} \mathrm{~cm}^{3}$.

At $T \sim 10^{4} \mathrm{~K}$, this is approximately $10^{5}$ times smaller than the expressions for $\Lambda_{\mathrm{H}}$ given above. Therefore, the cooling rate due to $\mathrm{H}-\mathrm{He}$ collisions becomes comparable to that due to $\mathrm{H}-\mathrm{e}^{-}$collisions only when the fractional ionization of the gas is very small, $x \sim 10^{-6}$. In the conditions relevant for $J_{\text {crit }}$, the fractional ionization is around a factor of 40 higher than this, and so it is safe to conclude that $\mathrm{H}-\mathrm{He}$ collisions make a negligible contribution to the total cooling rate.

\section{DISCUSSION}

\subsection{Combining the uncertainties}

It is useful at this point to draw a distinction between the uncertainties in $J_{\text {crit }}$ that exist due to the simplifications made in our chemical model and those that exist due to uncertainties in the chemical rate coefficient data. The case of $\mathrm{H}_{2}$ self-shielding provides a good example of the former type of uncertainty. As we have already discussed, the value of $J_{\text {crit }}$ that we recover in runs with a T5 spectrum is highly sensitive to our choice of $\mathrm{H}_{2}$ self-shielding function, increasing by more than a factor of five if we use the original Draine \& Bertoldi (1996) self-shielding function instead of the more recent version given in Wolcott-Green, Haiman 
Table 3. Error in $J_{\text {crit }}$ introduced by uncertainty in the rate coefficients of the listed reactions

\begin{tabular}{llcc}
\hline & & \multicolumn{2}{c}{ Uncertainty in $J_{\text {crit }}(\%)$} \\
No. & Reaction & T4 spec. & T5 spec. \\
\hline 2 & $\mathrm{H}_{2}+\mathrm{H} \rightarrow \mathrm{H}+\mathrm{H}+\mathrm{H}$ & 35 & 25 \\
3 & $\mathrm{H}^{-}+\mathrm{H} \rightarrow \mathrm{H}_{2}+\mathrm{e}^{-}$ & 40 & 40 \\
6 & $\mathrm{H}+\mathrm{e}^{-} \rightarrow \mathrm{H}^{-}+\gamma$ & 20 & 30 \\
10 & $\mathrm{H}+\mathrm{H} \rightarrow \mathrm{H}^{+}+\mathrm{e}^{-}+\mathrm{H}$ & 80 & 120 \\
11 & $\mathrm{H}^{-}+\mathrm{H} \rightarrow \mathrm{H}+\mathrm{H}+\mathrm{e}^{-}$ & Negligible & 60 \\
\hline
\end{tabular}

\& Bryan (2011). However, this large uncertainty ultimately stems from the fact that these self-shielding functions are both approximations, valid in different limits. In reality, it is likely that neither alternative provides a completely accurate description of the behaviour of the $\mathrm{H}_{2}$ in the gravitationally collapsing gas over the whole range of number densities of interest. Since the molecular data needed to compute the $\mathrm{H}_{2}$ photodissociation rate and self-shielding function is known accurately, we can in principle eliminate this uncertainty simply by using a more sophisticated treatment of the $\mathrm{H}_{2}$ in our simulation that tracks the population of the different rotational and vibrational levels and computes the photodissociation rate separately for each level. Similarly, any uncertainties in the $\mathrm{H}_{2}$ photodissociation rate that are introduced by the simple approximations commonly used to compute $N_{\mathrm{H}_{2}}$ can be eliminated by abandoning these approximations using a more accurate approach to compute the $\mathrm{H}_{2}$ column density (see e.g. Wolcott-Green, Haiman \& Bryan 2011; Hartwig et al. 2015).

Uncertainties in $J_{\text {crit }}$ that exist due to uncertainties in the chemical rate coefficient data are less easily dealt with. In order to reduce their impact, it is necessary to determine the rate coefficients more accurately, either experimentally or computationally, but this can be an extremely challenging and time-consuming occupation. It is therefore useful to assess the size of the uncertainty in $J_{\text {crit }}$ that results from these rate coefficient uncertainties, so that we can better assess how important it is to work on reducing these.

From the discussions in Sections 3 and 4 it is clear that in runs performed with a T4 spectrum, there are only four chemical reactions that introduce significant uncertainties into $J_{\text {crit }}$ : the collisional dissociation of $\mathrm{H}_{2}$ (reaction 2), the associative detachment of $\mathrm{H}^{-}$to form $\mathrm{H}_{2}$ (reaction 3 ), the formation of $\mathrm{H}^{-}$by radiative association (reaction 6) and the collisional ionization of $\mathrm{H}$ by $\mathrm{H}$ (reaction 10 ). These reactions individually introduce uncertainties of at least $25 \%$ into $J_{\text {crit }}$, as summarized in Table 3 , while the uncertainties contributed by the other processes discussed in Sections 3 and 4 are at the level of $10 \%$ or less. In runs with a T5 spectrum, the uncertainties introduced by these four reactions remain important, but $J_{\text {crit }}$ also depends strongly on the rate of reaction 11 , the collisional detachment of $\mathrm{H}^{-}$by collisions with $\mathrm{H}$.

We can place some bounds on the total uncertainty introduced into $J_{\text {crit }}$ by these reactions by deliberately choosing rate coefficients from amongst the different possibilities that either maximize or minimize $J_{\text {crit }}$. If we do this, then we find that in runs with a T4 spectrum, $J_{\text {crit,min }}=9.8$ and $J_{\text {crit, } \max }=47.5$. In runs with a T5 spectrum, we find instead that $J_{\text {crit,min }}=850$ and $J_{\text {crit, } \max }=5540$. Therefore, in the worst case, chemical rate coefficient uncertainties could introduce an uncertainty of around a factor of five into our estimates of $J_{\text {crit }}$.

In practice, the situation is probably not as bad as this, as the uncertainties in the different rate coefficients will cancel with each other to some extent. To assess the importance of this, we recalculated $J_{\text {crit }}$ a dozen times for both the $\mathrm{T} 4$ and $\mathrm{T} 5$ cases, in each calculation using rate coefficients for reactions $2,3,6,10$ and 11 that were randomly selected from amongst the different possibilities 4 This procedure gave us 12 different values of $J_{\text {crit }}$ for each choice of spectrum, and we then calculated the mean and standard deviation of these sets of values. We found that for runs with a T4 spectrum, $J_{\text {crit }}=21 \pm 5$, while for runs with a $\mathrm{T} 5$ spectrum, $J_{\text {crit }}=2400 \pm 700$. We can therefore conclude that the total uncertainty in $J_{\text {crit }}$ due to uncertainties in the chemical rate coefficients probably does not exceed a factor of two and certainly does not exceed a factor of five.

For comparison, determinations of $J_{\text {crit }}$ made using $3 \mathrm{D}$ simulations rather than the simplified one-zone approach used here typically find that $J_{\text {crit }}$ varies by at least a factor of a few from halo to halo owing to minor differences in the details of the collapse of the gas and its resulting temperature structure (Shang, Bryan \& Haiman 2010, Latif et al. 2014). Even larger uncertainties are introduced if we abandon the use of our simplified $\mathrm{T} 4$ and $\mathrm{T} 5$ spectra and consider more realistic models for the interstellar radiation field (Sugimura, Omukai \& Inoue 2014, Agarwal \& Khochfar 2015 Agarwal et al. 2015), particularly once we account for the fact that there may be a non-negligible X-ray component (Inayoshi \& Tanaka 2015; Latif et al. 2015). Therefore, it seems clear from the results of our study that astrophysical uncertainties have a larger impact on $J_{\text {crit }}$ than chemical uncertainties.

\subsection{Comparison with previous work}

Previous studies of the direct collapse model using a onezone approach have recovered a range of different values for $J_{\text {crit }}$, with values ranging from $18-39$ in runs with a T4 spectrum, and 1400-16000 in runs with a T5 spectrum (see the overviews of previous work in Inayoshi \& Tanaka 2015 and Agarwal et al. 2015). Some of this uncertainty, particularly in the T5 runs, is due to differences in the treatment of $\mathrm{H}_{2}$ self-shielding (Sugimura, Omukai \& Inoue 2014), and some can be ascribed to differences in the composition of the chemical networks used to model the gas, as explored in Paper I. However, it is also useful to assess how much of this uncertainty might reasonably be due to differences in the chemical rate coefficients used within these different models.

Specifically, in this section we compare the results that we obtain using our standard choices for the various rate coefficients with those that we obtain using the set of rate coefficients adopted within (a) the ENZO primordial chemistry

\footnotetext{
${ }^{4}$ In the case of reactions 2 and 3, we accounted for the $25 \%$ systematic uncertainty in the two rate coefficients by considering both our fiducial versions and also variants that were 1.25 and 0.75 times these fiducial versions.
} 
Table 4. Values of $J_{\text {crit }}$ derived using the ENZO and KROME primordial chemistry networks

\begin{tabular}{lcc}
\hline \multirow{2}{*}{ Model } & \multicolumn{2}{c}{$J_{\text {crit }}$} \\
& T4 spec. & T5 spec. \\
\hline \multirow{2}{*}{ ENZO (unmodified) } & 34.0 & 3050 \\
ENZO (modified) & 24.1 & 2480 \\
KROME (unmodified) & 8.2 & 690 \\
KROME (modified) & 11.2 & 1080 \\
\hline
\end{tabular}

network (Bryan et al. 2014), and (b) the KROME astrochemistry package (Grassi et al. 2014). We choose to compare our results with these two alternative treatments because many of the previous studies of direct collapse use one or the other of them, and moreover it is easy to gather information on exactly which rate coefficients are used within these models.

\subsubsection{The ENZO network}

Direct comparison between the results we obtain with our chemical network and those obtained using the ENZO primordial chemistry network is complicated by the fact that the ENZO network not only makes different choices for a number of the chemical and cooling rate coefficients, but also consists of a different set of chemical reactions from those included in our model. In particular, the chemical network implemented in the most recent version of the ENZO code (version 2.4) omits two processes that have a significant influence on the value of $J_{\text {crit }}$ : the collisional ionization of $\mathrm{H}$ by $\mathrm{H}$ (reaction 10) and the dissociative tunneling contribution to the $\mathrm{H}_{2}$ collisional dissociation rate. Therefore, to allow us to distinguish between the differences in $J_{\text {crit }}$ caused by the omission of these reactions and the differences in $J_{\text {crit }}$ caused by the differences in the rate coefficients, we compare our results with those from two additional sets of calculations. In one, we use the unmodified ENZO network. In the other, we use a version of this network that we have modified to include the two omitted processes mentioned above.

The results of our comparison are shown in Table 4. We see that the ENZO network systematically produces higher values of $J_{\text {crit }}$ that those derived using our network. With the unmodified ENZO network, $J_{\text {crit }}$ is increased by almost a factor of two for both the T4 and the T5 spectrum. With the modified network, on the other hand, the increase is smaller: around $30 \%$ for the T4 spectrum and $50 \%$ for the T5 spectrum. Note that these values were computing using the case A hydrogen recombination rate, which is the default choice in ENZO. If we instead use the case B rate, which is more physically appropriate in the present case, we recover values of $J_{\text {crit }}$ that are almost a factor of two larger than those in Table 4.

The highest value quoted in the literature for $J_{\text {crit }}$ in the case of a T4 spectrum and a one-zone calculation comes from Shang, Bryan \& Haiman (2010), who find that $J_{\text {crit }}=39$, roughly a factor of two larger than most other estimates. However, they use the ENZO chemistry network in their study, and our results here suggest that much of this factor of two differences is due to the composition of their network and their choice of reaction rate coefficients. It therefore seems plausible that most of the scatter in the reported val- ues of $J_{\text {crit }}$ for the T4 spectrum comes from differences in the chemical models used for the different studies.

\subsubsection{The KROME package}

As already noted in Paper I, the KROME package provides a number of different networks suitable for simulating primordial gas, but provides little guidance regarding which network should be used for which application. For studying direct collapse, the most suitable choice appears to be the react_xrays network. We therefore use this as the basis of our comparison $5^{5}$ As in the case of the ENzO network, this omits the effects of the collisional ionization of $\mathrm{H}$ by $\mathrm{H}$, and so when carrying out our comparison, we consider both the unmodified version of the react_xrays network plus a modified version that includes this reaction.

Our results are shown in Table 4 We see that the unmodified network underestimates $J_{\text {crit }}$ by around a factor of two. Including the collisional ionization of $\mathrm{H}$ by $\mathrm{H}$ improves the situation slightly, but even in this case the KROME network still significantly underestimates $J_{\text {crit }}$. We have investigated the causes of this discrepancy and find that it is largely driven by the choice of $\mathrm{H}^{+}$recombination rate. The KROME react_xrays network adopts the case A rate for this process, which causes the $\mathrm{H}^{+}$abundance to fall off more rapidly with increasing density than in our models. If we instead use the more physically appropriate case $B$ rate, we find much better agreement between the KROME results and our own determination of $J_{\text {crit }}$. This demonstrates that the error introduced into $J_{\text {crit }}$ is solely a consequence of the particular choice of network and rate coefficients, and not any intrinsic problem within the KROME infrastructure itself - if KROME is used simply as an ODE solver and cooling function, with a user-supplied chemical network that includes all of the important chemical reactions and an up-to-date set of rate coefficients, then we expect it to be able to produce exactly the same values of $J_{\text {crit }}$ as we find in our study.

\section{SUMMARY}

In this paper, we have attempted to determine the extent to which estimates of $J_{\text {crit }}$ are affected by uncertainties in the chemical rate coefficients and cooling rates used to model the chemical and thermal evolution of primordial gas. To do this, we first examined how sensitive $J_{\text {crit }}$ is to variation in the rate coefficients of the 26 reactions included in the reduced chemical network of Glover (2015). For those reactions where the sensitivity is large, we then critically examined the information available regarding the values of their rate coefficients, and quantified the effect of uncertainties in these values on our derived value of $J_{\text {crit }}$. We also carried out a similar analysis for the main cooling processes included in our model.

We found that there are five key chemical reactions where the combination of sensitivity and uncertainty leads to an appreciable $(>20 \%)$ uncertainty in $J_{\text {crit }}$. These are

\footnotetext{
5 Specifically, we consider the version present in the main KROME repository on March 26th, 2015, at which time the most recent commit was cf3d6b882d35545657d0a5daa7965b5c62c7e970
} 
the collisional dissociation of $\mathrm{H}_{2}$ by $\mathrm{H}$ (reaction 2), the formation of $\mathrm{H}_{2}$ by associative detachment of $\mathrm{H}^{-}$(reaction 3 ), the formation of $\mathrm{H}^{-}$by radiative association (reaction 6), the collisional ionization of $\mathrm{H}$ by $\mathrm{H}$ (reaction 10) and the collisional detachment of $\mathrm{H}^{-}$by $\mathrm{H}$ (reaction 11), although the last of these is important only in situations where $\mathrm{H}^{-}$ photodetachment is unimportant. Amongst these reactions, the single largest uncertainty comes from the collisional ionization of $\mathrm{H}$ by $\mathrm{H}$, a process which is very poorly constrained at the low energies relevant for this study (Barklem|2007). In addition, in gas illuminated with a hard T5 spectrum, $J_{\text {crit }}$ is also highly sensitive to the value of the $\mathrm{H}_{2}$ photodissociation rate, although in this case the intrinsic uncertainty in the rate is small, and the main difficulty comes from the fact that computationally efficient treatments of $\mathrm{H}_{2}$ self-shielding are often highly over-simplified (see e.g. Wolcott-Green \& Haiman 2011). The total uncertainty introduced into $J_{\text {crit }}$ by uncertainties in the remaining 20 reactions is small in comparison to the effects of these six reactions. Similarly, the remaining uncertainties in the cooling rates of $\mathrm{H}_{2}$ and atomic hydrogen do not significantly affect $J_{\text {crit }}$.

In the unlikely event that the errors in the different rate coefficients combine so as to maximum or minimize $J_{\text {crit }}$, we find that for a T4 spectrum, $J_{\text {crit,min }}=9.8$ and $J_{\text {crit, } \max }=47.5$, while for a T5 spectrum, $J_{\text {crit, } \min }=850$ and $J_{\text {crit, } \max }=5540$. Therefore, in the worst case, chemical rate coefficient uncertainties could introduce an uncertainty of around a factor of five into our estimates of $J_{\text {crit }}$. In the more likely case that the errors in the different rate coefficients are uncorrelated, we find that $J_{\text {crit }}$ probably lies in the range $J_{\text {crit }}=21 \pm 5$ for a T 4 spectrum and $J_{\text {crit }}=2400 \pm 700$ for a $\mathrm{T} 5$ spectrum.

Finally, we have compared the values of $J_{\text {crit }}$ that we have derived using our reaction network and rate coefficients with those that we obtain if we use instead the network and rate coefficients from (a) the ENzO hydrodynamical code or (b) the KROME astrochemistry package, both of which have been used in a number of previous studies of the direct collapse model. We find that the ENZO chemical model tends to overestimate $J_{\text {crit }}$ by around a factor of two, although this discrepancy increases to a factor of four if the physically appropriate case B hydrogen recombination rate is used in place of the case $A$ rate that is the default in ENzO. The KROME model, on the other hand, underestimates $J_{\text {crit }}$ by around a factor of two.

\section{ACKNOWLEDGEMENTS}

The author would like to thank the anonymous referee for their detailed report on an earlier version of this paper, and for calculating the rate coefficient for $\mathrm{H}-\mathrm{H}$ collisional ionization quoted in Equation 14. The author would also like to thank B. Agarwal and B. Smith for prompting him to think about this problem in the first place. Financial support for this work was provided by the Deutsche Forschungsgemeinschaft via SFB 881, "The Milky Way System" (sub-projects B1, B2 and B8) and SPP 1573, "Physics of the Interstellar Medium" (grant number GL 668/2-1), and by the European Research Council under the European Community's Seventh Framework Programme (FP7/2007-2013) via the ERC Advanced Grant STARLIGHT (project number 339177).

\section{REFERENCES}

Abel, T., Anninos, P., Zhang, Y., \& Norman, M. L. 1997, New Astron., 2, 181

Abgrall, H., \& Roueff, E. 1989, A\&AS, 79, 313

Agarwal, B., Khochfar, S., Johnson, J. L., Neistein, E., Dalla Vecchia, C., \& Livio, M. 2012, MNRAS, 425, 2854

Agarwal, B., \& Khochfar, S. 2015, MNRAS, 446, 160

Agarwal, B., Smith, B., Glover, S. C. O., Khochfar, S., \& Natarajan, P., 2015, MNRAS, in prep.

Ahn, K., Shapiro, P. R., Iliev, I. T., Mellema, G., \& Pen, U.-L. 2009, ApJ, 695, 1430

Bardsley, J. N., Herzenberg, A., \& Mandl, F. 1966a, Proc. Phys. Soc., 89, 305

Bardsley, J. N., Herzenberg, A., \& Mandl, F. 1966b, Proc. Phys. Soc., 89, 321

Barklem, P. S. 2007, A\&A, 466, 327

Barklem, P. S., Belyaev, A. K., Guitou, M., Feautrier, N., Gadéa, F. X., \& Spielfiedel, A. 2011, A\&A, 530, A94

Begelman, M. C., Volonteri, M., \& Rees, M. J. 2006, MNRAS, 370, 289

Begelman, M. C. 2010, MNRAS, 402, 673

Black, J. H. 1981, MNRAS, 197, 553

Boothroyd, A. I., Martin, P. G., Keogh, W. J., \& Peterson, M. R. 1991, J. Chem. Phys., 95, 4343

Boothroyd, A. I., Keogh, W. J., Martin, P. G., \& Peterson, M. R. 1996, J. Chem. Phys., 104, 7139

Bray, I., \& Stelbovics, A. T. 1993, Phys. Rev. Lett., 70, 746

Bromm, V., \& Loeb, A. 2003, ApJ, 596, 34

Browne, J. C., \& Dalgarno, A. 1969, J. Phys. B, 2, 885

Bryan, G. L., et al. 2014, ApJS, 211, 19

Cen, R. 1992, ApJS, 78, 341

Chen, J. C. Y., \& Peacher, J. L. 1968, Phys. Rev., 167, 30

Cízek, M., Horácek, J., \& Domcke, W. 1998, J. Phys. B, 31,2571

Clark, P. C., Glover, S. C. O., \& Klessen, R. S. 2012, MNRAS, 420, 745

Coppola, C. M., Longo, S., Capitelli, M., Palla, F., \& Galli, D. 2011, ApJS, 193, 7

Croft, H., Dickinson, A. S., \& Gadea, F. X. 1999, MNRAS, 304, 327

Dalgarno, A., \& Lepp, S. 1987, in Astrochemistry, ed. M. S. Vardya \& S. P. Tarafdar (Dordrecht: Reidel), 109

de Jong, T. 1972, A\&A, 20, 263

Dijkstra, M., Haiman, Z., Mesinger, A., Wyithe, J. S. B. 2008, MNRAS, 391, 1961

Draine, B. T., \& Bertoldi, F. 1996, ApJ, 468, 269

Drawin, H.-W., 1968, Zeit. Phys. 211, 404

Drawin, H.-W., 1969, Zeit. Phys. 225, 470

Esposito, F., \& Capitelli, M. 2009, J. Phys. Chem. A, 113, 15307

Ferland, G. J., Peterson, B. M., Horne, K., Welsh, W. F., \& Nahar, S. N. 1992, ApJ, 387, 95

Forrey, R. C., Balakrishnan, N., Dalgarno, A., \& Lepp, S. 1997, ApJ, 489, 1000

Fussen, D., \& Kubach, C. 1986, J. Phys. B, 19, L31

Galli, D., \& Palla, F., 1998, A\&A, 335, 403

Gealy, M. W., \& van Zyl, B. 1987, Phys. Rev. A, 36, 3100

Gerlich, D., Jusko, P., Roučka, Š., Zymak, I., Plašil, R., \& Glosík, J. 2012, ApJ, 749, 22

Glover, S. C. O., 2011, Habilitation thesis, Univ. Heidelberg

Glover, S. C. O., 2015, MNRAS, 451, 2082 (Paper I) 
Glover, S. C. O., \& Abel, T. 2008, MNRAS, 388, 1627 Glover, S. C., Savin, D. W., \& Jappsen, A.-K. 2006, ApJ, 640,553

Glover, S. C. O., \& Savin, D. W. 2009, MNRAS, 393, 911

Grassi, T., Bovino, S., Schleicher, D. R. G., Prieto, J., Seifried, D., Simoncini, E., \& Gianturco, F. A. 2014, MNRAS, 439, 2386

Greif, T. H., \& Bromm, V. 2006, MNRAS, 373, 128

Grosser, J., \& Krüger, W. 1984, Z. Phys. A, 318, 25

Gryzinski, M., 1965, Phys. Rev. A, 138, 336

Haiman, Z., Abel, T., \& Rees, M. J. 2000, ApJ, 534, 11

Hartwig, T., Glover, S. C. O., Klessen, R. S., Latif, M. A., \& Volonteri, M. 2015, MNRAS, 452, 1233

Hill, J., Geddes, J., \& Gilbody, H. B. 1979, J. Phys. B, 12, 3341

Hollenbach, D., \& McKee, C. F. 1979, ApJS, 41, 555

Hollenbach, D., \& McKee, C. F. 1989, ApJ, 342, 306

Hui, L., \& Gnedin, N. Y. 1997, MNRAS, 292, 27

Hutchins, J. B., 1976, ApJ, 205, 103

Inayoshi, K., \& Omukai, K. 2011, MNRAS, 416, 2748

Inayoshi, K., \& Tanaka, T. L. 2015, MNRAS, 450, 4350

Janev, R. K., Langer, W. D., \& Evans, K. 1987, Elementary Processes in Hydrogen-Helium plasmas (Berlin: Springer) Janev, R. K., Reiter, D., \& Samm, U. 2003, Berichte des Forschungszentrums Jülich, 4105

Kreckel, H., Bruhns, H., Č́žzek, M., Glover, S. C. O., Miller, K. A., Urbain, X., \& Savin, D. W. 2010, Science, 329, 69 Kunc, J. A., \& Soon, W. H. 1991, J. Chem. Phys., 95. 5738 Latif, M. A., Bovino, S., Van Borm, C., Grassi, T., Schleicher, D. R. G., \& Spaans, M. 2014, MNRAS, 443, 1979

Latif, M. A., Bovino, S., Grassi, T., Schleicher, D. R. G., \& Spaans, M. 2015, MNRAS, 446, 3163

Launay, J. M., Le Dourneuf, M., \& Zeippen, C. J. 1991, A\&A, 252, 842

Lenzuni, P., Chernoff, D. F., \& Salpeter, E. E. 1991, ApJS, 76, 759

Lepp, S., Buch, V., \& Dalgarno, A. 1995, ApJS, 98, 345

Liu, B. 1973, J. Chem. Phys., 58, 1925

Lotz, W. 1967, ApJS, 14, 207

Mandy, M. E., \& Martin, P. G. 1993, ApJS, 86, 199

Martin, P. G., Keogh, W. J., \& Mandy, M. E. 1998, ApJ, 499, 793

Martin, P. G., Schwarz, D. H., \& Mandy, M. E. 1996, ApJ, 461, 265

McClure, G. W. 1968, Phys. Rev., 166, 22

Mielke, S. L., Garrett, B. C., \& Peterson, K. A. 2002, J. Chem. Phys., 116, 4142

Miyake, S., Stancil, P. C., Sadeghpour, H. R., Dalgarno, A., McLaughlin, B. M., \& Forrey, R. C. 2010, ApJ, 709, L168

Moseley, J., Aberth, W., \& Peterson, J. R. 1970, Phys. Rev. Lett. 24, 435

Omukai, K., 2001, ApJ, 546, 635

Ramaker, D. E., \& Peek, J. M. 1976, Phys. Rev. A, 13, 58

Riley, M. E., \& Burke, R. A. 1999, J. Phys., B, 32, 5279

Schleicher, D. R. G., Palla, F., Galli, D., \& Latif, M. 2013, A\&A, 558, A59

Schleicher, D. R. G., Spaans, M., \& Glover, S. C. O. 2010, ApJ, 712, L69

Scholz, T. T., \& Walters, H. R. J. 1991, ApJ, 380, 302

Shah, M. B., Elliott, D. S., \& Gilbody, H. B. 1987, J. Phys. B, 20, 3501
Shang, C., Bryan, G. L., \& Haiman, Z. 2010, MNRAS, 402, 1249

Shapiro, P. R., \& Kang, H. 1987, ApJ, 318, 32

Shingal, R., Bransden, B. H., \& Flower, D. R. 1989, J. Phys., B, 22, 855

Shull, J. M. 1978, ApJ, 219, 877

Siegbahn, P., \& Liu, B. 1978, J. Chem. Phys., 68, 2457

Soon, W. H. 1992, ApJ, 394, 717

Stancil, P. C., Babb, J. F., \& Dalgarno, A. 1993, ApJ, 414, 672

Stancil, P. C., Lepp, S., \& Dalgarno, A. 1998, ApJ, 509, 1 Stenrup, M., Larson, A., \& Elander, N. 2009, Phys. Rev. A, 79, 012713

Sugimura, K., Omukai, K., \& Inoue, A. K. 2014, MNRAS, 445,544

Sun, Y., \& Dalgarno, A. 1994, ApJ, 427, 1053

Tanaka, T., \& Haiman, Z. 2009, ApJ, 696, 1798

Truhlar, D. G., \& Horowitz, C. J. 1978, J. Chem. Phys., 68,2466

Urbain, X., Lecointre, J., Mezdari, F., Miller, K. A., \& Savin, D. W. 2012, J. Phys. Conf. Ser., 388, 092004

Varandas, A. J. C., Brown, F. B., Mead, C. A., Truhlar, D. G., \& Blais, N. C. 1987, J. Chem. Phys., 86, 6258

Wishart, A. W., 1979, MNRAS, 187, P59

Wolcott-Green, J., \& Haiman, Z. 2011, MNRAS, 412, 2603

Wolcott-Green, J., Haiman, Z., \& Bryan, G. L. 2011, MNRAS, 418, 838

Wolniewicz, L., Simbotin, I., \& Dalgarno, A. 1998, ApJS, 115, 293

Wrathmall, S. A., \& Flower, D. R. 2007, J. Phys. B, 40, 3221

Wrathmall, S. A., Gusdorf, A., \& Flower, D. R. 2007, MNRAS, 382, 133 